\title{
Dissociation of S-R Compatibility and Simon Effects With Mixed Tasks and Mappings
}

\author{
Robert W. Proctor and Motonori Yamaguchi \\ Purdue University
}

\author{
Varun Dutt and Cleotilde Gonzalez \\ Carnegie Mellon University
}

\begin{abstract}
Binary-choice reactions are typically faster when the stimulus location corresponds with that of the response than when it does not. This advantage of spatial correspondence is known as the stimulusresponse compatibility (SRC) effect when the mapping of stimulus location, as the relevant stimulus dimension, is varied to be compatible or incompatible with response location. It is called the Simon effect when stimulus location is task-irrelevant. The SRC effect is eliminated when compatible and incompatible spatial mappings are mixed within a trial block, and the Simon effect is eliminated when the Simon task is mixed with the SRC task with incompatible spatial mapping. Eliminations of both types have been attributed to suppression of an automatic response-activation route. We tested predictions of this suppression hypothesis for conditions in which the SRC and Simon tasks were intermixed and the spatial mappings on the SRC trials could be compatible or incompatible. In Experiment 1, the two tasks were equally likely, as were compatible and incompatible spatial mappings on SRC trials; in Experiment 2, the SRC or Simon task was more frequent; and, in Experiment 3, the compatible or incompatible location mapping for the SRC task was more frequent. The SRC effect was absent overall in all experiments, whereas the Simon effect was robust to the manipulations and showed the characteristic decrease across the reaction time (RT) distribution. This dissociation of effects implies that the automatic responseactivation route is not suppressed in mixed conditions and suggests that mixing influences the SRC and Simon effects by different means.
\end{abstract}

Keywords: dual-route account, mixed tasks, Simon effect, stimulus-response compatibility, task switching

A standard finding for binary choice-reaction tasks, in which people respond to whether a stimulus occurs in a left or right location with a left or right keypress, is that reaction time (RT) is shorter when the stimulus locations are assigned to their corresponding responses (compatible mapping) than when they are assigned to the opposite responses (incompatible mapping). This advantage for the compatible mapping is called the spatial stimulus-response compatibility (SRC) effect, and it is a variant of the display-control compatibility effects that influence performance in many contexts (see Proctor \& Vu, 2006, for a review). A similar, though smaller, advantage of spatial correspondence oc-

This article was published Online First September 10, 2012.

Robert W. Proctor and Motonori Yamaguchi, Department of Psychological Sciences, Purdue University; Varun Dutt and Cleotilde Gonzalez, Dynamic Decision Making Laboratory, Department of Social and Decision Sciences, Carnegie Mellon University.

Motonori Yamaguchi is now at Department of Psychology, Vanderbilt University. Varun Dutt is now at the School of Computing and Electrical Engineering and School of Humanities and Social Sciences, Indian Institute of Technology, Mandi, India.

The research described in the present paper was supported in part by ARO MURI grant W911NF-05-1-0153.

Correspondence concerning this article should be addressed to Robert W. Proctor, Department of Psychological Sciences, Purdue University, 703 Third St., West Lafayette, IN 47907-2081. E-mail: proctor@ psych.purdue.edu curs for tasks in which a nonspatial stimulus attribute such as color is relevant and stimulus location is irrelevant. This stimulusresponse (S-R) correspondence effect based on task-irrelevant stimulus location is known as the Simon effect (Simon, 1990). SRC and Simon effects have been studied extensively in recent years (PsycINFO counts of 297 and 287 articles, respectively, from the year 2000 to July 7, 2012) because of the insight they provide into the processes that underlie selection among possible responses to stimulus events.

Both the SRC and Simon effects persist across hundreds of trials (e.g., Dutta \& Proctor, 1992; Proctor \& Lu, 1999) and are obtained for a variety of spatial stimulus and response sets in simple and complex tasks (Proctor \& Vu, 2006; Yamaguchi \& Proctor, 2006). However, the effects are absent or reversed when the SRC task is performed with compatible and incompatible trials mixed, or when trials from SRC and Simon tasks are mixed (Proctor \& Vu, 2002a). Similar explanations in terms of suppression of a direct responseactivation route have been proposed for the impact of mixed mappings and mixed tasks on the SRC and Simon effects, but logical differences between the two mixing procedures and some empirical results suggest that the procedures are not comparable. Prior studies have investigated each type of mixing separately (e.g., Vu \& Proctor, 2008), but unique predictions of existing accounts can be tested when both types of mixing occur within a single trial block. Thus, in the present study we measured the joint influences of mixed spatial mappings and tasks on the SRC and Simon effects. 


\section{Dual-Route Models}

The SRC and Simon effects are customarily attributed to correspondence between spatial stimulus codes and response codes (e.g., Proctor \& Vu, 2006). Debate has centered on whether the spatial stimulus code is a result of a shift of attention to the stimulus (e.g., Rubichi, Nicoletti, Iani, \& Umiltà, 1997) or of relations of the stimulus to various reference frames (e.g., Yamaguchi \& Proctor, 2012). The spatial response codes are often credited to the spatial nature of the required response discrimination (e.g., Ansorge \& Wühr, 2004; Wühr \& Ansorge, 2007). Hommel (2011) recently summarized the current state of understanding as follows:

The human brain seems to code all sorts of spatial aspects of a stimulus event and there is evidence that all these codes can interact with the spatial representation of an action directed to, or at least triggered by a given stimulus (p. 193).

Many results involving activation of response codes with respect to the SRC and Simon effects are consistent with dual-route models of response selection (e.g., Kornblum, Hasbroucq, \& Osman, 1990; Spironelli, Tagliabue, \& Umiltà, 2009). According to these models, activation of response codes can occur by way of indirect (or controlled) and direct (or automatic) routes. The indirect route produces activation of the response codes to the relevant stimulus feature through $\mathrm{S}-\mathrm{R}$ associations or rules defined by the task instructions (often called short-term S-R associations, because they are applicable only to the current task). In contrast, the direct route yields activation of the spatially corresponding response code by way of long-term associations (e.g., Zorzi \& Umiltà, 1995) or similarity (Wühr \& Ansorge, 2007). The Simon effect is attributed to this direct activation of the corresponding response, which facilitates performance when that response is correct but interferes when it is incorrect. The SRC effect, too, is often credited in part to activation of the corresponding response via the direct route, as in Kornblum et al.'s model, but also to differences in time for response activation to occur by way of the short-term location associations of the indirect route. Indeed, some authors (e.g., Fitts \& Deininger, 1954) have attributed the SRC effect entirely to "S-R translations" of the indirect route.

When the RT distribution is divided into quantiles, or bins, and the Simon effect is plotted as a function of bin, the effect is largest at the shortest RTs and then decreases as RT lengthens (De Jong, Liang, \& Lauber, 1994; see Proctor, Miles, \& Baroni, 2011, for a review). This pattern is suggestive of rapid activation of the corresponding response through the long-term associations of the direct route, which then dissipates, possibly due to intentional inhibition of the initial activation (e.g., Ridderinkhof, 2002). Unlike the Simon effect, the SRC effect increases across the RT distribution (Vu \& Proctor, 2008), consistent with the view that the SRC effect does not have its basis entirely in direct activation of the corresponding response.

\section{Mixing Mappings and Tasks}

As noted, the SRC effect can be eliminated by mixing trials with compatible and incompatible spatial mappings. Shaffer (1965) had participants respond to a left or right stimulus light with a left or right keypress. On each trial, simultaneous with onset of the light, a mapping signal appeared that specified the mapping for that trial (horizontal line for compatible; vertical line for incompatible). RT was longer for the blocks in which the mappings were mixed than for blocks in which all trials had the same mapping. More important, this increase of RT under mixed conditions was greater for the compatible mapping than for the incompatible mapping, resulting in no SRC effect. Instead of a 54-ms benefit for the compatible mapping in unmixed conditions, there was no significant difference $(-8 \mathrm{~ms})$ in the mixed conditions.

This outcome has been replicated in several studies that used green/red color of the left or right imperative stimulus (Heister \& Schroeder-Heister, 1994; Vu \& Proctor, 2004, 2008, 2011; Yamaguchi \& Proctor, 2006) or low/high tone pitch (De Jong, 1995) as the mapping signal, indicating that it is not dependent on how the mapping is cued. The favored account has been that the direct response-activation route is proactively suppressed when mapping uncertainty exists (e.g., De Jong, 1995; Vu \& Proctor, 2004). However, Vu and Proctor $(2008,2011)$ found the SRC effect under mixed mapping conditions to be similar to that of pure mapping conditions early in the RT distribution, but then to decrease (and become negative) at longer RTs, rather than increasing as for the pure mapping conditions. That the reduction in SRC effect caused by mixed mappings occurs mainly later in the RT distribution rather than earlier suggests that proactive suppression of the direct route may not be responsible for the reduction.

The SRC effect is also eliminated when the location-relevant trials of an SRC task, with only one mapping, are mixed with location-irrelevant trials of a Simon task. Again, color of the left or right stimulus has usually been used to signal the task, with white indicating that location is relevant and red or green specifying color as relevant. In this case, the SRC task is performed no faster when the spatial mapping is compatible than when it is incompatible (Proctor \& Vu, 2002b; Proctor, Vu, \& Marble, 2003). Too, the mapping in force for the SRC task influences the Simon effect for those trials on which stimulus location is irrelevant: With compatible mapping, the Simon effect is increased in size; with incompatible mapping, the Simon effect reverses to favor the spatially noncorresponding response (Marble \& Proctor, 2000; Proctor et al., 2003). This reversal has been taken to suggest that the longterm associations of the direct route are suppressed and contributing little to performance.

\section{Sequential Effects}

The relative contributions of the direct route (long-term associations) and indirect route (short-term associations) to performance have also been studied by examining sequential effects. In the SRC task with mixed compatible and incompatible mappings, the mapping can repeat or switch from one trial to the next. The SRC effect tends to be positive for trials on which the mapping repeats, but it reverses to favor the incompatible mapping for trials on which the mapping switches (Shaffer, 1965; Vu \& Proctor, 2008, 2011). Sequential effects have also been studied for pure Simon tasks, in which all trials are location-irrelevant. When the Simon effect is analyzed as a function of whether the prior trial was corresponding or noncorresponding, the effect is large after corresponding trials and absent after noncorresponding trials (e.g., Iani, Rubichi, Gherri, \& Nicoletti, 2009; Stürmer, Leuthold, Soetens, Schröter, \& Sommer, 2002). 
This absence is most often attributed to reactive suppression (or inhibition) of the direct route as a result of conflict being detected on a noncorresponding trial, with the inhibition released after a corresponding trial (e.g., Iani et al., 2009; Stürmer et al., 2002; but see Hommel, Proctor, \& Vu, 2004, and Wendt, Kluwe, \& Peters, 2006, for a different explanation). The reactive inhibition account predicts that sequential effects should not interact with variables that influence the Simon effect through the short-term associations of the indirect route (e.g., Iani et al., 2009). Contrary to this prediction, Soetens, Maetens, and Zeischka (2010) found that mixed SRC trials acted mainly to increase the Simon effect after a corresponding trial when the mapping for the SRC task was compatible, but to reverse the Simon effect after a noncorresponding trial when the mapping was incompatible.

\section{Present Study}

In addition to the SRC and Simon tasks individually differing in terms of whether stimulus location is relevant or irrelevant, there are several distinctions between the mixed-task and mixedmapping procedures (see Vu \& Proctor, 2008). In the mixed-task procedure, a single spatial mapping is in effect during a trial block, and the uncertainty is about whether stimulus location or another feature (often color) will be relevant. Selection is of which stimulus feature (location or color) should determine responding, a process that demands attention and takes time (Proctor \& Fisicaro, 1977). Conversely, in the mixed-mapping procedure, stimulus location is always relevant, and a decision must be made on each trial as to which spatial mapping is appropriate. This uncertainty about mapping is what prevents a rapid response for trials on which the correct response is compatible. In the present study, we tested situations for which there was uncertainty about both which task to perform (i.e., respond to stimulus location or color) and, for the SRC task, which mapping to apply. This mixture of task uncertainty and mapping uncertainty enabled us to address several theoretical issues regarding the influence of mixing on performance.

In Experiment 1, we used a procedure for which stimulus location was relevant on half of the trials (SRC task, with equal proportions of compatible and incompatible mappings) and stimulus color was relevant on the other half (Simon task, with green color mapped to one response and red to the other). According to the suppression hypothesis, the direct route should be proactively suppressed in this dual-mixing context as it is when either tasks or mappings alone are mixed. For the SRC task, the prediction is that the SRC effect will be absent, with the RT distribution function for the effect being relatively flat (because there is no activation of the corresponding response). In contrast, if the corresponding response is activated initially and then inhibited (e.g., Ridderinkhof, 2002) or application of the compatible-mapping rule in the indirect route has been blocked (De Jong, 1995), an SRC effect should be evident early in the distribution function but decrease at longer RTs (i.e., the function should have a negative slope). For the Simon task, the short-term location associations established for the SRC task should not bias the corresponding or noncorresponding spatial relation because the compatible and incompatible location mappings are equally likely. Consequently, if the direct route is proactively suppressed, there should be no Simon effect, positive or negative. However, if the long-term associations of the direct route are not suppressed, lack of bias from the short-term associations should allow a positive Simon effect of typical size to emerge, which decreases across the RT distribution.

In Experiment 2, task expectancy was varied by having one task occur on $80 \%$ of the trials and the other on $20 \%$ for half of the participants and reversing this relation for the other half. Thus, half of the participants were biased to expect stimulus location to be relevant and half to expect stimulus color to be relevant. According to the proactive suppression account, in the condition biased toward the SRC task, suppression of the direct route should still occur, much like when location is relevant for all trials and spatial mappings are mixed, resulting in an absence of SRC and Simon effects. However, in the condition with bias toward the Simon task, participants should prepare much as they would for a pure Simon task, that is, without suppressing the direct route, in which case the Simon and SRC effects would both be expected to occur.

In Experiment 3, the two tasks occurred equally often, but the priority of the compatible and incompatible mappings for the SRC task was varied by making $80 \%$ of the SRC trials compatible and $20 \%$ incompatible for half of the participants, and vice versa for the other half. This manipulation should bias participants to prepare for the dominant spatial mapping, resulting in a positive SRC effect when the compatible mapping is most frequent and a negative SRC effect when the incompatible mapping is most frequent. If the short-term associations for the compatible and incompatible mappings maintained for the SRC task contribute equally to performance for trials on which stimulus location is irrelevant, then the Simon effect should be of similar size regardless of which mapping predominates. However, if the difference in mapping frequencies for the SRC task strengthens the associations for the predominant spatial relation compared with the subordinate one, then the results for the Simon task should be like those obtained when only a single SRC mapping is in effect: The Simon effect should be positive when the majority of the SRC trials are congruent and negative when the majority are incongruent.

For all experiments, we also examined sequential effect patterns. These patterns allow evaluation of whether the influence of correspondence repetition/switch (whether the stimulus and response locations on successive trials have the same or different correspondence relation) on the SRC and Simon effects is independent of any influence of task repetition/switch, and whether these influences are in agreement with what would be predicted on the basis of trial-to-trial modulation of suppression of the direct response-selection route.

\section{Experiment 1}

Experiment 1 was similar to prior ones in which SRC and Simon tasks were mixed (e.g., Marble \& Proctor, 2000) in that trials from each task occurred randomly, with equal frequency. However, whereas in prior studies the spatial mapping for the SRC task was constant for each participant, in the present case the mapping for the SRC task was compatible on half of the trials and incompatible on the other half. Thus, $25 \%$ of trials were SRC with compatible mapping, 25\% were SRC with incompatible mapping, and 50\% were Simon (color relevant, with stimulus and response locations corresponding on half of the trials). We chose to signal the task by color, as in previous studies, with white designating location as relevant (SRC task) and red or green designating color as relevant 
(Simon task). To signal the mapping on SRC trials, we chose to use a separate horizontal or vertical line, as in Shaffer's (1965) study. As noted, prior mixed mapping studies have shown this line-orientation signal to yield similar results as when a color of the imperative stimulus is used to signal mapping (e.g., $\mathrm{Vu} \&$ Proctor, 2004), which would have been too confusing in the present case because red or green color was relevant for the Simon task.

Because the SRC effect is eliminated when tasks are mixed, as well as when mappings are mixed, we expected that there would be no benefit for the compatible mapping in the SRC task. According to the suppression hypothesis, this absence of the SRC effect is attributable to proactive suppression of the direct route or longterm associations (Proctor \& Vu, 2002a). If this account is correct, then there should be no Simon effect because the corresponding response would not be receiving activation from the long-term associations of the direct route (which would be suppressed). At the same time, because both compatible and incompatible mappings for the SRC task are equally likely, the corresponding response would not be receiving activation from the short-term location associations in effect for the SRC task either (whose activation should not be biased toward either response). If, instead, the long-term associations are not being suppressed under mixedtask conditions, then this removal of bias for the short-term associations (compared with prior studies in which all of the SRC trials had the same mapping) should let the Simon effect emerge. Thus, a positive Simon effect of normal magnitude would imply that the long-term associations of the indirect route continue to be active when SRC and Simon tasks are mixed.

\section{Method}

Participants. Thirty-two students (20 males, 8 females, 4 of unspecified gender) at Purdue University, recruited from the introductory psychology courses, participated for experiment credits. All participants in this and subsequent experiments were required to have normal or corrected-to-normal visual acuity and normal color vision, as indicated by self-report.

Apparatus and stimuli. The apparatus consisted of a personal computer and 17-in. VGA monitor. The experiment was controlled by a custom computer application programmed in Visual Basic 6.0. The imperative stimulus was a green, red, or white filled circle ( $8 \mathrm{~mm}$ diameter) presented on the left or right side of the screen $(6.5 \mathrm{~cm}$ from screen center). Green and red circles specified the location-irrelevant trials (the Simon task), and the white circle specified the location-relevant trials (the SRC task). On the latter trials, a mapping signal (a white horizontal or vertical line of 1.5 $\mathrm{mm}$, centered on the screen) was shown simultaneously with the imperative stimulus; no mapping signal was presented on the Simon trials. Responses were made by pressing a left (' $z$ ') or right (' $/$ ') key on the computer keyboard with the left and right index fingers, respectively. RT was the interval between onset of an imperative stimulus and depression of a response key.

Task and procedure. The experiment was conducted individually for each participant in a dimly lit room. The participant sat in front of the computer monitor, at a viewing distance of approximately $55 \mathrm{~cm}$, and read instructions displayed on the screen. The instructions emphasized that participants were to make responses as fast and as accurately as possible.
Participants performed mixed tasks, responding according to the color of the imperative stimulus on some trials (the Simon task) and to its location on others (the SRC task). For the Simon task, half the participants used one color-response mapping, and half used the other. For the SRC task, a horizontal or vertical mapping signal was presented simultaneously with the imperative stimulus (a white circle on the left or right). For half of the participants, the horizontal signal required them to press the response key on the same side as the location of the imperative stimulus (the compatible mapping) and the vertical cue the response key on the opposite side (the incompatible mapping). For the other half of the participants, the signal-mapping relation was reversed. Each participant performed four blocks of 160 trials, half of the trials with the Simon task and half the SRC task. The total number of trials was 320 for the Simon task and 320 for the SRC task (160 trials with the compatible mapping and 160 trials with the incompatible mapping). The two types of trials appeared equally often in each block in a random order. Participants were not informed as to the proportions of particular trial conditions.

An experimental session started with a block of 32 practice trials, 16 of the Simon task and 16 of the SRC task, intermixed. Participants were allowed to repeat the practice block as many times as they wanted, so that they were sufficiently familiar with the task requirements. No participant performed more than three practice blocks.

Each trial started with a white fixation cross (consisting of vertical and horizontal lines, $5 \mathrm{~mm}$ long) on the center of screen for $500 \mathrm{~ms}$. After a 500-ms blank display, the imperative stimulus occurred on the left or right of the screen, with a mapping signal provided only on the SRC trials. The imperative stimulus was presented until a response was made or for 1,500 $\mathrm{ms}$ if no response was made. When participants pressed an incorrect key, an error tone was presented from external speakers placed on the sides of the computer monitor. The tone's frequency was $400 \mathrm{~Hz}$, and its duration was $500 \mathrm{~ms}$. If a response was correct, a 500-ms blank screen was displayed which equated the intertrial interval for correct and incorrect responses. A trial ended with a 1-s blank screen. An experimental session lasted less than an hour.

\section{Results}

Trials for which RT was $<100 \mathrm{~ms}$ were considered anticipations, and trials for which RT was $>1,500 \mathrm{~ms}$ were recorded as no response. These trials were not included in the analyses. To examine sequential effects, trials that immediately followed error trials were excluded. Together with anticipation and no-response trials, the proportion of discarded trials was 5.4\%. Mean RT for correct responses and percentage error (PE) for the SRC task were analyzed as a function of mapping (compatible, incompatible) on the current trial, task sequence (whether the task on the current trial was a repetition or switch of the task from the preceding trial), and correspondence sequence (whether the correspondence relation between stimuli and responses on the present trial was a repetition or switch of the correspondence relation from the preceding trial). The results for the SRC task are summarized in Table 1. The Simon task was analyzed similarly, but with correspondence (corresponding, noncorresponding) replacing mapping as a factor, and its results are also summarized in Table 1. The SRC and Simon effects, as a function of task sequence and correspondence se- 
Table 1

Experiment 1: Mean Response Time (in ms) and Percentage Error as a Function of Task Sequence and Correspondence Sequence for the Compatible (Comp) and Incompatible (Incomp) Mappings of the SRC Task and the Corresponding (Corr) and Noncorresponding (Noncorr) Trials of the Simon Task

\begin{tabular}{|c|c|c|c|c|c|}
\hline \multirow[b]{2}{*}{ Task sequence } & \multirow{2}{*}{$\begin{array}{l}\text { Correspondence } \\
\text { sequence }\end{array}$} & \multicolumn{2}{|c|}{ Response time } & \multicolumn{2}{|c|}{ Percentage error } \\
\hline & & Comp & Incomp & Comp & Incomp \\
\hline \multicolumn{6}{|l|}{ SRC task } \\
\hline \multirow[t]{2}{*}{ Repeat } & Repeat & 566 & 577 & 1.63 & 1.67 \\
\hline & Switch & 676 & 652 & 9.82 & 5.86 \\
\hline \multirow[t]{3}{*}{ Switch } & Repeat & 664 & 672 & 4.58 & 4.30 \\
\hline & Switch & 691 & 675 & 6.22 & 4.19 \\
\hline & & Corr & Noncorr & Corr & Noncorr \\
\hline \multicolumn{6}{|l|}{ Simon task } \\
\hline \multirow[t]{2}{*}{ Repeat } & Repeat & 539 & 559 & 0.88 & 2.40 \\
\hline & Switch & 598 & 591 & 3.98 & 4.61 \\
\hline \multirow[t]{2}{*}{ Switch } & Repeat & 647 & 670 & 5.90 & 7.50 \\
\hline & Switch & 666 & 674 & 5.35 & 7.46 \\
\hline
\end{tabular}

quence, are shown in Figure 1. For all within-subject terms of the reported analyses of variance (ANOVAs), the degrees of freedom are from the original ANOVA but the $p$ values are those for the Huynh-Feldt correction for violations of sphericity, except where noted.

\section{SRC task.}

$\boldsymbol{R T}$ analysis. The SRC effect was absent overall: The main effect of mapping was not significant, $F(1,31)<1$, with the compatible and incompatible mappings showing similar RTs ( $M \mathrm{~s}=649$ and $644 \mathrm{~ms}$, respectively). The only interaction involving mapping was that with correspondence sequence (see Figure

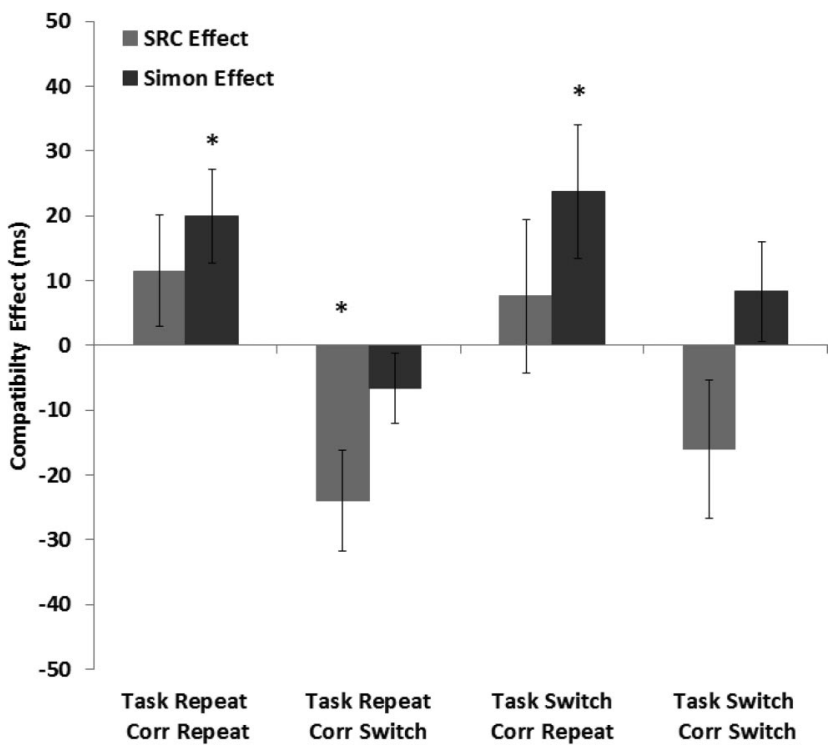

Figure 1. The stimulus-response compatibility (SRC) effect and Simon effect as a function of task sequence and correspondence sequence in Experiment 1 (error bars represent one standard error of means; asterisks indicate significant effects at $\alpha=.05$, as indicated by paired-sample $t$ tests at each data point).
$1), F(1,31)=26.43, M S E=528, p<.001$. The SRC effect was $12 \mathrm{~ms}$ when the correspondence relation was the same on the prior trial as on the current trial, $t(31)=1.07, S E=8.90, p>.20$, but it was $-20 \mathrm{~ms}$ when the correspondence relation on the prior trial differed from that on the current trial, $t(31)=2.46, S E=8.14, p<$ .020 .

There were main effects of task sequence, $F(1,31)=144.61$, $M S E=1,471, p<.001$, and correspondence sequence, $F(1,31)=$ $114.97, M S E=1,619, p<.001$. Responses were faster when the task repeated (i.e., the prior trial was another SRC trial; $M=618$ $\mathrm{ms}$ ) than when it switched (i.e., the prior trial was a Simon trial; $M=675 \mathrm{~ms}$ ), and when the correspondence relation repeated $(M=620 \mathrm{~ms})$ than when it switched $(M=674 \mathrm{~ms})$. The two factors also interacted, $F(1,31)=83.46, M S E=1,134, p<.001$. Repeating the correspondence relation yielded a larger advantage when location had been relevant on the prior trial (i.e., it was an SRC trial; $M=92 \mathrm{~ms}$ ) than when location had been irrelevant (i.e., it was a Simon trial, $M=15 \mathrm{~ms}$ ).

Analysis of the SRC effect across the RT distribution was performed by rank ordering the RTs for compatible trials and incompatible trials separately for each participant, dividing them into five bins (quintiles), and obtaining a mean RT value for each participant in each bin (see Proctor et al., 2011, for a review of the method). The data, plotted in Figure 2, were submitted to a two-factor repeated-measures ANOVA involving bins and mapping. Of concern was the interaction term, which was significant, $F(4,124)=3.79$, $M S E=454, p<.031:$ The SRC effect decreased $28 \mathrm{~ms}$ from the first $\mathrm{RT}$ bin $(11 \mathrm{~ms})$ to the last bin $(-17 \mathrm{~ms})$.

$\boldsymbol{P E}$ analysis. Unlike the RT data, the main effect of mapping was significant, $F(1,31)=5.83, M S E=26.27, p<.022$, but it reflected a negative SRC effect $(M=-1.56 \%)$, with PE less for the incompatible mapping than for the compatible mapping. Mapping interacted with correspondence sequence, $F(1,31)=7.23$, $M S E=18.25, p<.011$, with the negative SRC effect being smaller when the S-R mapping repeated $(-0.12 \%)$ than when it switched $(-2.99 \%)$

The main effect of correspondence sequence was also significant, $F(1,31)=44.92, M S E=17.21, p<.001$, but that of task sequence was not, $F(1,31)<1$. However, the interaction of these

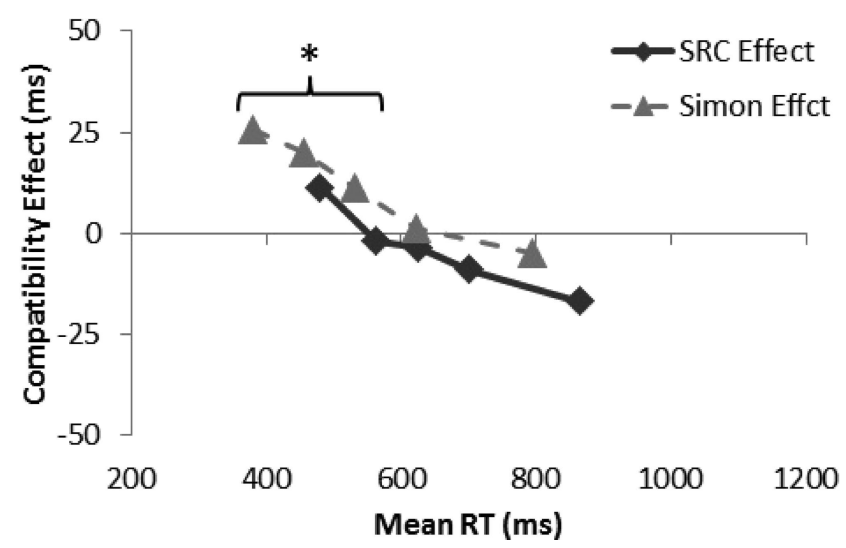

Figure 2. Plots of the stimulus-response compatibility (SRC) effect and the Simon effect against mean response times in the quintile bins in Experiment 1 (effects for the bracketed points, with asterisk, were significant at $\alpha=.05$, as indicated by paired-sample $t$ tests at each data point) 
two factors was significant, $F(1,31)=22.45, M S E=20.93, p<$ .001. As with RT, the advantage of repeating the correspondence relation was larger when the previous trial was of the SRC task $(M=6.19 \%)$ than when it was of the Simon task $(M=0.77 \%)$.

\section{Simon task.}

RT analysis. There was a main effect of correspondence, $F(1$, $31)=5.39, M S E=1525, p<.027$, indicating an overall $11-\mathrm{ms}$ Simon effect, and correspondence interacted with correspondence sequence, $F(1,31)=6.35, M S E=1113, p<.017$. The Simon effect was $22 \mathrm{~ms}$ when the correspondence relation repeated and 1 ms when it switched (see Figure 1).

The main effects of task sequence, $F(1,31)=85.70, M S E=$ $6,381, p<.001$, and correspondence sequence, $F(1,31)=61.85$, $M S E=860, p<.001$, were significant, and the two variables interacted, $F(1,31)=32.97, M S E=563, p<.001$. As for the $\mathrm{SRC}$ task, RT was shorter when the task repeated $(M=572 \mathrm{~ms})$ than when it switched $(M=664 \mathrm{~ms})$, and when the correspondence relation repeated $(M=604 \mathrm{~ms})$ than when it switched $(M=$ $633 \mathrm{~ms})$. The advantage of repeating rather than switching the correspondence relation was larger when the previous trial was a Simon trial $(M=46 \mathrm{~ms})$ than when it was an $\operatorname{SRC}$ trial $(M=$ $12 \mathrm{~ms}$ ).

Because RT data for the Simon task are often analyzed as a function of correspondence on the prior trial, correspondence on the current trial, and task sequence, we conducted an additional sequential analysis of this type. For it, the three-way interaction among all factors was significant, $F(1,31)=32.97, M S E=563$, $p<.001$. When the task switched (i.e., the prior trial was from the SRC task), the Simon effect was $28 \mathrm{~ms}$ if the current trial followed a compatible-mapping trial and $4 \mathrm{~ms}$ if it followed an incompatible-mapping trial, consistent with the suppression account. When the task repeated (i.e., the prior trial was from the Simon task), this result pattern was amplified: The Simon effect was $53 \mathrm{~ms}$ if the prior trial was corresponding and $-39 \mathrm{~ms}$ if it was noncorresponding. This reversal seems contrary to an account solely in terms of the direct route being reactively inhibited after a trial for which there is response conflict.

An RT distribution analysis of the Simon effect similar to that for the SRC effect showed a Bin $\times$ Correspondence interaction (see Figure 2), $F(4,124)=11.63, M S E=223, p<.001$. The Simon effect decreased by $31 \mathrm{~ms}$ from the first RT bin $(26 \mathrm{~ms})$ to the last bin $(-5 \mathrm{~ms})$.

$\boldsymbol{P E}$ analysis. There was a main effect of correspondence, $F(1$, $31)=6.50, M S E=26.85, p<.016$. The Simon effect was $1.65 \%$. The main effect of task sequence was also significant, $F(1,31)=$ $31.43, M S E=37.22, p<.001$, and that variable interacted with correspondence sequence, $F(1,31)=4.60, M S E=16.29, p<$ .040 . PE was less when the task repeated $(M=2.28 \%)$ than when it switched $(M=6.56 \%)$, and the advantage of repeating the correspondence relation was $1.87 \%$ when the previous trial was also a Simon trial and $-0.29 \%$ when it was an SRC trial.

\section{Discussion}

The RT data showed no overall benefit for the compatible mapping over the incompatible mapping in the SRC task, with the $\mathrm{PE}$ data in fact showing a significant cost. This result is in agreement with prior findings that the SRC effect is eliminated under conditions in which tasks or mappings are mixed. In con- trast, the Simon effect was significant in both the RT and PE data. We compared the Simon effect's size of $11 \mathrm{~ms}$ and $1.65 \%$ to that of a control group of 24 participants who performed only the Simon task. Their data showed a similar-sized Simon effect of 15 $\mathrm{ms}(M \mathrm{~s}=419$ and $433 \mathrm{~ms}$ for the corresponding and noncorresponding trials, respectively) and $1.02 \%(M \mathrm{~s}=3.52 \%$ and $4.54 \%$, respectively). ${ }^{1}$

That the Simon effect occurred when compatible and incompatible mappings for the SRC task were equally frequent, and therefore should not have produced any bias in short-term S-R associations toward either spatial relation, implies that the long-term associations of the direct route continue to activate the spatially compatible responses in mixed conditions. Consistent with this interpretation, the Simon effect showed its largest value of $26 \mathrm{~ms}$ at the first of five RT bins and then decreased to a slightly negative value at the last bin. Although the initial value of the SRC effect was only $11 \mathrm{~ms}$, it too decreased to a similar extent across the RT distribution (becoming negative; see Figure 2), as $\mathrm{Vu}$ and Proctor (2011) found when all trials were from the SRC task and only the mappings were mixed. This pattern suggests that the direct route may also be influencing performance of the SRC task and that the main impact of mixing is to prevent application of the compatible rule in the indirect response-activation route (e.g., De Jong, 1995)

Sequential analyses showed overall benefits for repetition of the task and of the correspondence relation, with the shortest RTs for the SRC and Simon tasks occurring when both types of repetition were present. Although there was no overall SRC effect in the RT data (and a negative one in the PE data), the SRC effect was more positive for trials on which the correspondence relation from the prior trial repeated than for those on which it switched, a pattern similar to that reported for mixed mapping versions of the SRC task (e.g., Shaffer, 1965; Vu \& Proctor, 2011). Likewise, the Simon effect was evident when the prior trial had the same correspondence relation as the current trial and absent when it did not. The influence of correspondence sequence on the SRC and Simon effects did not interact with whether the task repeated or switched, possibly because the conflict control mechanisms in each case involve the same spatial domain (e.g., Egner, 2008) Also, the Simon effect showed the usual pattern of being evident only when the prior trial was corresponding. The disparity in Simon effect size was larger when the prior trial was also from the Simon task, with a negative Simon effect being apparent when the

\footnotetext{
${ }^{1}$ Because all trials in the control condition were 'task-repeat' trials, we also compared the control condition to the task-repeat trials of the Simon task in Experiment 1, by submitting RT and PE to ANOVAs as a function of Correspondence, Correspondence Sequence, and Group. Significant effects involving the Group variable were its main effect, $F(1,54)=70.82$, $M S E=16,529, p<.001$, and $F(1,54)=9.41, M S E=17.89, p<.003$ for RT and PE, respectively, and the Correspondence Sequence $\times$ Group interaction, $F(1,54)=7.64, M S E=473, p<.008$, and $F(1,54)=8.59$ $M S E=5.27, p<.005$, for $\mathrm{RT}$ and $\mathrm{PE}$, respectively. Responses were generally faster but less accurate for the control group $(M=426 \mathrm{~ms}$, $4.03 \%)$ than for the task-repeat trials of Experiment $1(M=572 \mathrm{~ms}$, $2.28 \%)$. The advantage of mapping repetition was larger for the task-repeat trials of Experiment 1 ( $M=46 \mathrm{~ms})$ than for the control group $(M=30 \mathrm{~ms})$ for the RT data, but smaller for the former condition $(M=1.87 \%)$ than for the latter $(M=3.69 \%)$ for the PE data. Most important, there was no Correspondence $\times$ Group interaction in RT, $F(1,54)=1.74, M S E=487$, or $\mathrm{PE}, F(1,54)<1, M S E=12.86$.
} 
prior trial had a noncorresponding relation. This reversal argues against a reactive inhibition account of the sequential effects, implicating instead a bias to follow a noncorresponding response to stimulus color with another noncorresponding response.

\section{Experiment 2}

Manipulations of the percentages of trials are often used to bias participants' attention (e.g., He, Fan, Zhou, \& Chen, 2004) and response preparation (e.g., Moresi, Adam, Rijcken, \& Van Gerven, 2008). The idea is that participants will be biased to prepare for the predominant relation rather than the alternative one. In Experiment 2 , therefore, we directed participants' expectancies toward the SRC task or the Simon task by having one task performed on $80 \%$ of the trials and the other on only $20 \%$. When the SRC task occurs on $80 \%$ of the trials, participants should expect stimulus location to be relevant, much like in the studies of mixed compatible and incompatible mappings in which location is relevant on all trials. Consequently, no SRC effect is expected in the $80 \%$ SRC condition. If this absence of SRC effect is a result of suppression of the direct route, then the Simon effect should also be absent on the $20 \%$ of trials that are the Simon task. The logic for this prediction is similar to that of Experiment 1, but the present condition provides a stronger test of the suppression hypothesis because of the prevalence of the SRC trials.

When the Simon task occurs on $80 \%$ of the trials, participants should prepare for color to be relevant and stimulus location irrelevant, much as for a pure Simon task in which stimulus color is relevant on all trials. Thus, there should be less or no suppression of the direct response-activation route, and performance should be better when the irrelevant stimulus location corresponds with the response, that is, a Simon effect should be obtained. If the direct route is not suppressed in this case, and such suppression contributes to elimination of the SRC effect under mixed-task conditions, then the SRC effect should reappear as well.

\section{Method}

Participants. Forty-eight new students (mostly-Simon group: 15 males and 9 females; mostly-SRC group: 17 males and 7 females) from the same pool as in Experiment 1 participated.

Apparatus, stimuli, and procedure. The apparatus consisted of a 19-in. LCD computer monitor, and the experiment was controlled by the same computer program as in the preceding experiment. Stimuli were identical with those used in Experiment 1. The procedure also followed closely that of Experiment 1. All participants performed the practice phase in the same way as in Experiment 1, for which the Simon and SRC trials occurred equally often. In the test phase of the present experiment, however, the frequency of trials was manipulated. For half the participants (mostly-SRC group), $80 \%$ of trials were those of the SRC task (where the compatible- and incompatible-mapping trials occurred equally often), and $20 \%$ of trials were those of the Simon task. For the other half (mostly-Simon group), $80 \%$ of trials were those of the Simon task, and the remaining $20 \%$ were those of the SRC task. The instructions were similar to those of Experiment 1 and did not specify that there would be unequal percentages of each trial type. As shown in the second paragraph that follows, though, participants traded off performance on the respective tasks in a manner in accord with their relative frequencies.

\section{Results}

Trials were filtered in the same manner as in Experiment 1, which excluded $4.53 \%$ of the trials. Mean RT for correct responses and PE were computed for the SRC and Simon tasks as a function of the same factors as in Experiment 1, with group (mostly-SRC, mostly-Simon) as an additional factor (see Table 2). The SRC and Simon effects are summarized in Figure 3.

An initial test was done to validate that participants traded off performance on the two tasks in accord with the percentage manipulation. For this test, mean RT and PE were calculated for each participant for each of the two tasks, and task (SRC; Simon) was entered as a variable along with group in a two-factor ANOVA. The Task $\times$ Group interaction was significant for both measures, $F \mathrm{~s}(1,46)=15.23$ and 4.94, MSE $=1,842$ and 12.94, $p \mathrm{~s}<.001$ and .035. For the mostly-Simon group, RT was $92 \mathrm{~ms}$ longer for the SRC task than for the Simon task $(M \mathrm{~s}=728$ and $636 \mathrm{~ms})$ and PE was $2.0 \%$ greater $(M \mathrm{~s}=5.90 \%$ and $3.90 \%)$, whereas for the mostly-SRC group, these differences were $24 \mathrm{~ms}(M \mathrm{~s}=687$ and $663 \mathrm{~ms}$ for the SRC and Simon tasks) and $-1.27 \%(M \mathrm{~s}=4.32 \%$ and $5.59 \%$, respectively). Thus, the manipulation of relative frequencies of the respective tasks induced the intended tradeoff between the tasks.

\section{SRC task.}

RT analysis. In contrast to Experiment 1, the main effect of mapping was significant, $F(1,46)=4.39, M S E=3,262, p<.042$, but its interaction with correspondence sequence was not, $F(1,46)=2.86, M S E=1,211, p=.10$. The SRC effect was slightly negative overall $(M=-12 \mathrm{~ms})$, showing a benefit for the incompatible mapping, with the reversal tending to be less when the correspondence relation on the current trial was the same as that on the previous trial $(M=-6 \mathrm{~ms})$ than when it was different ( $M=-18 \mathrm{~ms}$ ). This pattern is qualitatively similar to that shown in Experiment 1 and is inconsistent with the prediction of the suppression account.

There were significant main effects of task sequence, $F(1,46)=$ 273.80, $M S E=2,330, p<.001$, and correspondence sequence, $F(1,46)=59.51, M S E=1,524, p<.001$, and the two factors interacted, $F(1,46)=63.62, M S E=1,363, p<.001$. These results were similar to those of Experiment 1, with the advantage of repeating a corresponding or noncorresponding spatial relation being larger when the previous trial was an SRC trial $(M=61 \mathrm{~ms})$ than when it was a Simon trial $(M=1 \mathrm{~ms})$. Of note, no interaction involving group was significant.

An RT distribution analysis like that performed in Experiment 1 (see Figure 4), but with group as an additional between-subjects factor, showed marginally significant interactions of Bin $\times$ Compatibility and of those two variables with group, $F \mathrm{~s}(4,184)=2.38$ and $2.68, M S E=411, p s=.053$ and .033 with sphericity assumed and .096 and .072 with the Huynh-Feldt adjustment. The SRC effect tended to become more negative across the RT distribution, with the change being greater for the mostly-SRC group (SRC effects of $5,-2,-8,-16$, and $-26 \mathrm{~ms}$ across the five RT bins) than for the mostly-Simon group (SRC effects of $-8,-12,-15$, -15 , and $-5 \mathrm{~ms}$ ).

$\boldsymbol{P E}$ analysis. Mapping again showed a main effect, $F(1$, 46) $=6.92, M S E=58.59, p<.012$, and there was an inter- 
Table 2

Experiment 2: Mean Response Time (in ms) and Percentage Error of the Mostly-Simon and Mostly-SRC Groups as a Function of Task Sequence and Correspondence Sequence for the Compatible (Comp) and Incompatible (Incomp) Mappings of the SRC Task and the Corresponding (Corr) and Noncorresponding (Noncorr) Trials of the Simon Task

\begin{tabular}{|c|c|c|c|c|c|}
\hline \multirow[b]{2}{*}{ Task sequence } & \multirow[b]{2}{*}{ Correspondence sequence } & \multicolumn{2}{|c|}{ Response time } & \multicolumn{2}{|c|}{ Percentage error } \\
\hline & & Comp & Incomp & Comp & Incomp \\
\hline \multicolumn{6}{|l|}{ SRC task } \\
\hline \multicolumn{6}{|c|}{ Mostly-Simon group } \\
\hline \multirow[t]{2}{*}{ Repeat } & Repeat & 662 & 663 & 9.14 & 6.55 \\
\hline & Switch & 729 & 704 & 5.02 & 6.60 \\
\hline \multirow[t]{2}{*}{ Switch } & Repeat & 771 & 768 & 11.04 & 4.91 \\
\hline & Switch & 766 & 758 & 2.75 & 1.18 \\
\hline \multicolumn{6}{|l|}{ Mostly-SRC group } \\
\hline \multirow[t]{2}{*}{ Repeat } & Repeat & 608 & 612 & 7.07 & 2.70 \\
\hline & Switch & 686 & 669 & 6.42 & 3.92 \\
\hline \multirow[t]{3}{*}{ Switch } & Repeat & 740 & 713 & 5.81 & 5.31 \\
\hline & Switch & 747 & 723 & 1.86 & 1.51 \\
\hline & & Corr & Noncorr & Corr & Noncorr \\
\hline \multicolumn{6}{|c|}{ Simon task } \\
\hline \multicolumn{6}{|c|}{ Mostly-Simon group } \\
\hline \multirow[t]{2}{*}{ Repeat } & Repeat & 529 & 557 & 4.76 & 7.11 \\
\hline & Switch & 579 & 588 & 5.30 & 5.40 \\
\hline \multirow[t]{2}{*}{ Switch } & Repeat & 699 & 722 & 3.30 & 2.91 \\
\hline & Switch & 694 & 717 & 0.93 & 1.49 \\
\hline \multicolumn{6}{|l|}{ Mostly-SRC group } \\
\hline \multirow[t]{2}{*}{ Repeat } & Repeat & 576 & 606 & 4.74 & 11.76 \\
\hline & Switch & 633 & 625 & 7.57 & 7.01 \\
\hline \multirow[t]{2}{*}{ Switch } & Repeat & 699 & 732 & 4.12 & 5.79 \\
\hline & Switch & 715 & 722 & 0.62 & 3.07 \\
\hline
\end{tabular}

action of Mapping $\times$ Correspondence Sequence, $F(1,46)=$ 5.26, MSE $=33.02, p<.026$. The SRC effect was negative, being $-3.40 \%$ when the S-R mapping repeated and $-0.71 \%$ when the mapping switched. For PE, there was a significant effect involving group, the three-way interaction of that factor with mapping and task sequence, $F(1,46)=10.51, M S E=$ 23.00, $p<.002$. For the mostly-Simon group, the SRC effect was $-0.51 \%$ when the previous trial was a Simon trial and $-3.85 \%$ when it was an SRC trial. For the mostly-SRC group, the SRC effect was- $3.44 \%$ when the previous trial was an SRC trial and $-0.43 \%$ when it was a Simon trial. In other words, the negative SRC effect for PE was larger when the previous trial was of the most frequent task than when it was of the less frequent task.
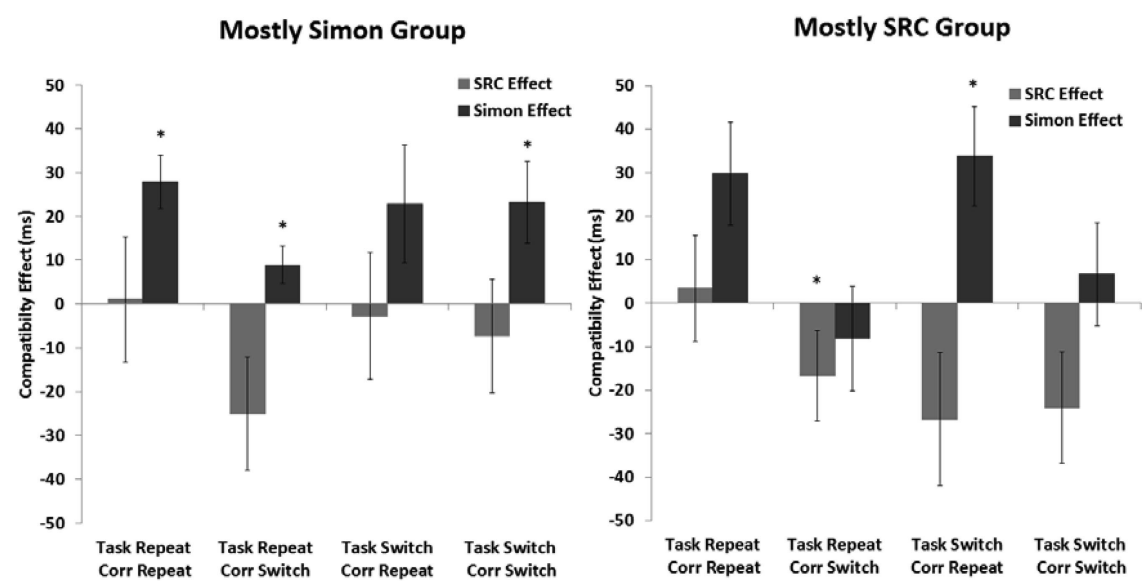

Figure 3. The stimulus-response compatibility (SRC) effect and the Simon effect as a function of task sequence and correspondence sequence for the mostly-Simon and mostly-SRC groups in Experiment 2 (error bars represent one standard error of means; asterisks indicate significant effects at $\alpha=.05$, as indicated by paired-sample $t$ tests at each data point). 


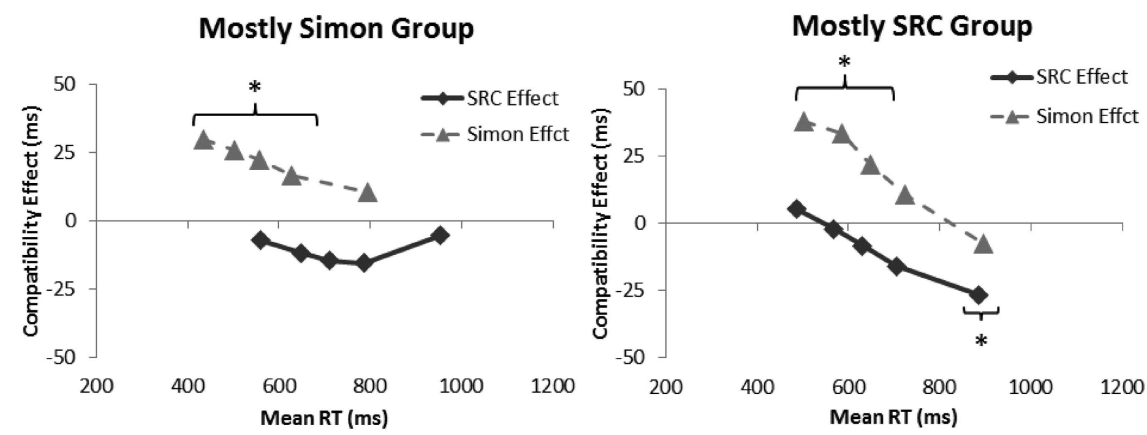

Figure 4. Plots of the stimulus-response compatibility (SRC) effect and the Simon effect against mean response times in the quintile bins in Experiment 2 (effects for the bracketed points, with asterisks, were significant at $\alpha=.05$, as indicated by paired-sample $t$ tests at each data point).

Otherwise, there were main effects of task sequence, $F(1,46)=$ 4.76, $M S E=53.60, p<.034$, and correspondence sequence, $F(1,46)=20.07, M S E=40.46, p<.001$, and they interacted, $F(1,46)=20.62, M S E=19.31, p<.001$. Again, these results were similar to those of Experiment 1 and, as for RT, the benefit of repeating the correspondence relation was larger when the prior trial was an SRC trial $(M=4.94 \%)$ than when it was a Simon trial $(M=0.87 \%)$.

\section{Simon task}

RT analysis. There was a main effect of correspondence, $F(1$, $46)=14.42, M S E=2,184, p<.001$, and that variable interacted with correspondence sequence, $F(1,46)=8.10, M S E=1,294$, $p<.007$. The Simon effect was $29 \mathrm{~ms}$ when the correspondence relation repeated and $8 \mathrm{~ms}$ when it switched, averaging $18 \mathrm{~ms}$ (see Figure 3).

The main effects of task sequence, $F(1,46)=219.21, M S E=$ $6,932, p<.001$, and correspondence sequence, $F(1,46)=29.67$, $M S E=1,185, p<.001$, were significant, as was their interaction, $F(1,46)=39.34, M S E=993, p<.001$. Again, the advantage of repeating the correspondence relation was larger when the previous trial was a Simon trial $(M=39 \mathrm{~ms})$ than when it was an SRC trial $(M=-1 \mathrm{~ms})$. In addition, task sequence interacted with group, $F(1,46)=4.91, M S E=6,932, p<.032$ : The cost of switching tasks was larger for the mostly-Simon group $(M=145$ $\mathrm{ms}$ ) than for the mostly-SRC group $(M=107 \mathrm{~ms})$. Group did not enter into any significant effect involving correspondence or correspondence sequence.

As in Experiment 1, we also analyzed the RT data for the Simon task as a function of correspondence on the prior trial, correspondence on the current trial, and task sequence, adding the variable of group. The three-way interaction of prior correspondence, current correspondence, and task sequence was again significant, $F(1$, 46) $=39.34, M S E=993, p<.001$, but those variables did not enter into a four-way interaction with group, $F(1,46)<1$. When the task switched, the Simon effect was $21 \mathrm{~ms}$ after a compatiblemapping trial and $23 \mathrm{~ms}$ after an incompatible-mapping trial. In contrast, when the task repeated, the Simon effect was $54 \mathrm{~ms}$ after a spatially corresponding trial compared with $-25 \mathrm{~ms}$ after a noncorresponding trial.

The RT distribution analysis (see Figure 4) showed a Bin $\times$ Correspondence interaction, $F(4,184)=7.86, M S E=517, p<$ .002 , but no three-way interaction with group, $F(4,184)=1.38$, $p=.255$. The Simon effect decreased across the distribution, much as in Experiment 1, from $34 \mathrm{~ms}$ at the first RT bin to $2 \mathrm{~ms}$ at the last bin.

$\boldsymbol{P E}$ analysis. PE showed main effects of correspondence, $F(1$, 46) $=6.49, M S E=40.18, p<.014$, correspondence sequence, $F(1,46)=5.49, M S E=46.79, p<.023$, and task sequence, $F(1$, $46)=37.30, M S E=39.71, p<.001$, and these three factors interacted, $F(1,46)=7.62, M S E=26.25, p<.008$. When the task repeated, the Simon effect was $4.69 \%$ if the correspondence relation repeated and $-0.24 \%$ if it switched. When the task switched, the effect was $0.65 \%$ if the correspondence relation repeated and $1.50 \%$ if it switched. No other effects were significant.

\section{Discussion}

The SRC effect was significantly negative in the present experiment, although the size was small. The normal advantage for the compatible mapping was absent not only when the SRC task predominated, as expected, but also when the Simon task predominated. This latter result is counter to the proactive suppression account because the direct route should not be suppressed when Simon trials predominate. The Simon effect again was apparent, and like the SRC effect, in the main ANOVA it did not enter significantly into any interaction with group for RT or PE. Moreover, the decrease in Simon effect across the RT distribution was similar for the mostly-SRC and mostly-Simon groups. That the Simon effect was equally apparent when SRC trials predominated, and the direct response-activation route should have been suppressed, again suggests that such proactive suppression was not occurring. The SRC effect also showed a negative slope across the RT distribution (although somewhat less so in the mostly-Simon group), which could be taken as another indication that the direct route was not suppressed. More generally, the results imply that the critical factor determining the overall SRC and Simon effects is mainly the task set defined by the location mappings for the SRC task and the color mappings for the Simon task, and not expectations about which task is more likely on a given trial.

As in Experiment 1, for both tasks, RT was shortest for trials on which the correspondence relation and task both repeated. Also, the Simon effect again was substantial after a correspondencerepeat trial and small after a correspondence-switch trial. This 
pattern did not interact with task, as in Experiment 1, or with group, being similar regardless of whether most trials were from the SRC task or Simon task. The SRC task likewise showed the pattern of a less negative SRC effect after a correspondence-repeat trial than after a correspondence-switch trial, as in Experiment 1, although this interaction was significant only in the PE data. This pattern also did not depend on task or group. Thus, Experiment 2 provides additional evidence that the mechanism producing the sequential dependencies for the Simon and SRC effects is not influenced much by task set and expectancies.

\section{Experiment 3}

In Experiment 3, the percentages of trials for the SRC and Simon tasks were held equal at $50 \%$, and the percentages of the compatible and incompatible mapping trials for the SRC task were varied. For one group, $80 \%$ of the SRC trials were compatible and $20 \%$ incompatible; for the other group the percentages were opposite. This manipulation introduces a bias in the location relation defined for the location-relevant trials rather than a bias in whether location or color will be relevant. This bias in location relation would be expected to yield an advantage for the compatible mapping when most SRC trials are compatible and an advantage for the incompatible mapping when most are incompatible. Any SRC effect beyond the influences of bias will be evident in the overall effect obtained by averaging across the two bias conditions.

What impact the mapping-bias manipulation would have on the Simon effect was of more interest. If the bias toward making the corresponding response on SRC trials is a result of the short-term location associations being stronger for the predominant relation than for the subordinate one, then a pattern of influence on the Simon effect similar to that on the SRC effect should occur: The Simon effect should be positive when compatible SRC trials predominate and negative when incompatible trials predominate. However, if the influence of mapping bias on the SRC task is not on the strength of the short-term location associations, then a Simon effect should be similarly evident when either compatible or incompatible SRC trials predominate because, being of similar strength, the influences of the compatible and incompatible shortterm associations that must be maintained to perform the task should average out.

\section{Method}

Participants. A new group of 48 undergraduate students (19 males and 5 females in the mostly compatible group; 19 males and 5 females in the mostly incompatible group) was recruited from the same participant pool as in the preceding experiments.

Apparatus, stimuli, and procedure. All aspects were identical to those of Experiment 2, except that the two tasks each occurred on $50 \%$ of the trials and the proportions of compatible and incompatible mapping trials of the SRC task were varied. For half the participants (mostly compatible group), $80 \%$ of the SRC trials were compatibly mapped and $20 \%$ were incompatibly mapped. For the other half (mostly incompatible group), the proportions were reversed. For the Simon task, the stimulus appeared equally often in a location that corresponded with that of the response and that did not correspond with it. Again, these relations were not stated in instructions, and the compatible and incompatible SRC trials appeared equally often during the practice phase.

\section{Results}

Trials were filtered in the same way as in Experiments 1 and 2, excluding $3.42 \%$ of the trials. Mean RT for correct responses and PE for the SRC and Simon tasks were computed as a function of the same factors as in Experiment 2, but with group being mostly compatible or mostly incompatible (see Table 3). The SRC and Simon effects are also summarized in Figure 5.

SRC task.

$\boldsymbol{R T}$ analysis. Mapping interacted with group, $F(1,46)=$ $78.31, M S E=4,584, p<.001$, with the bias manipulation having the expected impact of creating a benefit for the compatible mapping for the mostly compatible group $(40 \mathrm{~ms})$ but a cost for the mostly incompatible group $(-83 \mathrm{~ms})$. However, the main effect of mapping was also significant, $F(1,46)=9.61, M S E=4,584, p<$ .003 , indicating an overall SRC effect of $-22 \mathrm{~ms}$, that is, longer RT overall on compatible trials than on incompatible trials.

As in the prior experiments, there were main effects of task sequence, $F(1,46)=109.01, M S E=2,068, p<.001$, and correspondence sequence, $F(1,46)=272.74, M S E=1,653, p<$ .001 , and the two variables interacted, $F(1,46)=247.74, M S E=$ $1,524, p<.001$. The advantage of repeating the correspondence relation was larger when the previous trial was an SRC trial $(M=$ $131 \mathrm{~ms})$ than when it was a Simon trial $(M=6 \mathrm{~ms})$.

Group entered into several additional interactions, including a four-way interaction with the other three variables, $F(1,46)=$ $35.42, M S E=1,023, p<.001$. Consequently, separate ANOVAs were performed for each group. For the mostly compatible group, all terms were significant, $F(1,23)>15.80, p<.001$, except the two-way interaction of Task Sequence $\times$ Mapping, $F(1,23)=$ $2.08, p=.162$. When the task sequence repeated, there was a large benefit for the compatible mapping if the correspondence relation also repeated compared with a cost for the compatible mapping if the correspondence relation switched (see Figure 5). In contrast, when the task switched, the advantage for the compatible mapping was evident both when the correspondence relation repeated and when it switched. For the mostly incompatible group, all terms were significant, $F_{\mathrm{s}}(1,23)>7.61, p \mathrm{~s} \leq .011$. In this case, when the task repeated, the negative SRC effect (advantage for incompatible mapping) was larger when the correspondence relation also repeated than when it switched (see Figure 5), whereas when the task switched, the negative SRC effect did not depend on whether the correspondence relation repeated or switched.

In the RT distribution analysis (see Figure 6), the only interaction involving bin was that of Bin $\times$ Compatibility, $F(4,184)=$ 5.04, MSE $=473, p<.013$ : The SRC effect became progressively more negative, mainly across the first three RT bins, being -1 , $-17,-22,-24$, and $-25 \mathrm{~ms}$, respectively, when averaged across mapping emphasis groups.

$\boldsymbol{P E}$ analysis. There were main effects of correspondence sequence, $F(1,46)=33.83, M S E=51.13, p<.001$, and mapping, $F(1,46)=6.31, M S E=24.45, p<.001$, but not task sequence, $F(1,46)<1, M S E=17.12$. Task sequence interacted with group, $F(1,46)=10.93, M S E=17.12, p<.001$, correspondence sequence, $F(1,46)=26.53, M S E=22.41, p<$ .001 , and mapping, $F(1,46)=8.27, M S E=17.72, p<.001$. However, because there was a four-way interaction of Mapping $\times$ Correspondence Sequence $\times$ Task Sequence $\times$ Group, 
Table 3

Experiment 3: Mean Response Time (in ms) and Percentage Error of the Mostly-Compatible and Mostly-Incompatible Groups as a Function of Task Sequence and Correspondence Sequence for the Compatible (Comp) and Incompatible (Incomp) Mappings of the SRC Task and the Corresponding (Corr) and Noncorresponding (Noncorr) Trials of the Simon Task

\begin{tabular}{|c|c|c|c|c|c|}
\hline \multirow[b]{2}{*}{ Task sequence } & \multirow[b]{2}{*}{ Correspondence sequence } & \multicolumn{2}{|c|}{ Response time } & \multicolumn{2}{|c|}{ Percentage error } \\
\hline & & Comp & Incomp & Comp & Incomp \\
\hline \multicolumn{6}{|c|}{ SRC task } \\
\hline \multicolumn{6}{|c|}{ Mostly-Compatible group } \\
\hline \multirow[t]{2}{*}{ Repeat } & Repeat & 534 & 619 & 0.56 & 1.50 \\
\hline & Switch & 715 & 692 & 4.96 & 9.60 \\
\hline \multirow[t]{2}{*}{ Switch } & Repeat & 654 & 712 & 1.80 & 2.97 \\
\hline & Switch & 674 & 713 & 2.66 & 3.57 \\
\hline \multicolumn{6}{|c|}{ Mostly-Incompatible group } \\
\hline \multirow[t]{2}{*}{ Repeat } & Repeat & 613 & 520 & 1.67 & 0.61 \\
\hline & Switch & 718 & 686 & 7.70 & 3.06 \\
\hline \multirow[t]{3}{*}{ Switch } & Repeat & 733 & 633 & 8.18 & 1.30 \\
\hline & Switch & 737 & 631 & 7.17 & 1.94 \\
\hline & & Corr & Noncorr & Corr & Noncorr \\
\hline \multicolumn{6}{|c|}{ Simon task } \\
\hline \multicolumn{6}{|c|}{ Mostly-Compatible group } \\
\hline \multirow[t]{2}{*}{ Repeat } & Repeat & 577 & 621 & 0.83 & 1.38 \\
\hline & Switch & 630 & 644 & 1.46 & 4.32 \\
\hline \multirow[t]{2}{*}{ Switch } & Repeat & 683 & 712 & 4.04 & 3.58 \\
\hline & Switch & 697 & 708 & 2.30 & 9.20 \\
\hline \multicolumn{6}{|c|}{ Mostly-Incompatible group } \\
\hline \multirow[t]{2}{*}{ Repeat } & Repeat & 561 & 588 & 1.11 & 1.77 \\
\hline & Switch & 613 & 619 & 2.32 & 3.62 \\
\hline \multirow[t]{2}{*}{ Switch } & Repeat & 670 & 692 & 4.58 & 6.69 \\
\hline & Switch & 676 & 715 & 4.98 & 8.77 \\
\hline
\end{tabular}

$F(1,46)=7.79, M S E=16.27, p<.008$, we focus on separate analyses for each group.

For the mostly compatible group, all effects were significant, $F(1,23)>6.17, p<.025$, except for those of Task Sequence $\times$ Mapping, $F(1,23)=2.12, p=.159$, Correspondence Sequence $\times$ Mapping, $F(1,23)=2.50, p=.127$, and the three-way interaction of those variables, $F(1,23)=2.31, p=.142$. The only significant effect involving mapping was that the error rate was less overall when the mapping was compatible $(2.50 \%)$ than when it was incompatible $(4.41 \%)$. For the mostly incompatible group, the only nonsignificant effect was the Correspondence Sequence $\times$ Mapping interaction, $F(1,23)<1.00$; for the remaining terms, all $F \mathrm{~s}(1$, $23)>4.88, p<.05$. When the task repeated, the negative SRC effect was smaller if the correspondence relation repeated than if it switched (see Table 3); when the task switched, the SRC effect was larger if the correspondence relation repeated than if it switched.

\section{Simon task.}

$\boldsymbol{R T}$ analysis. There were main effects of task sequence, $F(1$, $46)=144.70, M S E=5,089, p<.001$, correspondence sequence, $F(1,46)=67.87, p<.001$, and correspondence, $F(1,46)=36.31$, $M S E=1,502, p<.001$. Correspondence sequence interacted with task sequence, $F(1,46)=25.64, M S E=818, p<.001$, and correspondence, $F(1,46)=6.33, M S E=653, p<.015$, and there was a three-way interaction of these factors, $F(1,46)=7.20$, $M S E=531, p<.010$. When the task repeated, the Simon effect was larger if the spatial correspondence relation repeated $(M=36$ $\mathrm{ms}$ ) than if it switched $(M=10 \mathrm{~ms})$; when the task switched, the
Simon effect was identical between these conditions $(M=25 \mathrm{~ms}$ for both). The remaining significant effects were two three-way interactions. That of Correspondence $\times$ Task Sequence $\times$ Group, $F(1,46)=4.85, M S E=670, p<.033$, reflects that the Simon effect tended to be larger when the task repeated than when it switched for the mostly compatible group (29 vs. $20 \mathrm{~ms}$ ) but smaller for the mostly incompatible group (16.5 vs. $30.5 \mathrm{~ms}$ ), although neither of these two-way interactions for each group was significant on its own, $F_{\mathrm{s}}(1,23)=1.70$ and $3.21, p \mathrm{~s}=.20$ and .087. The Correspondence $\times$ Correspondence Sequence $\times$ Group interaction, $F(1,46)=4.36, M S E=653, p<.042$, is attributable to the Simon effect being larger when the correspondence relation repeated than when it switched for the mostly compatible group (36.5 vs. $12.5 \mathrm{~ms}), F(1,23)=11.65, p<.002$, but not for the mostly incompatible group ( 24.5 vs. $22.5 \mathrm{~ms}), F(1,23)<1.0$.

Additional analysis in terms of correspondence on the preceding trial, correspondence on the current trial, task sequence, and group showed the three-way interaction of the variables other than group evident in Experiments 1 and 2, $F(1,46)=$ 25.64, $M S E=818, p<.001$, but group did not enter into a four-way interaction with those variables, $F(1,46)<1, M S E=$ 818, as in Experiment 2. When the task switched, the Simon effect was $35 \mathrm{~ms}$ if the current trial followed a compatiblemapping trial and $15 \mathrm{~ms}$ if the current trial followed an incompatible-mapping trial. When the task repeated, the Simon effect was $62 \mathrm{~ms}$ if the current trial followed a spatially corresponding trial and $-17 \mathrm{~ms}$ if the current trial followed a noncorresponding trial. 
Mostly Compatible Group

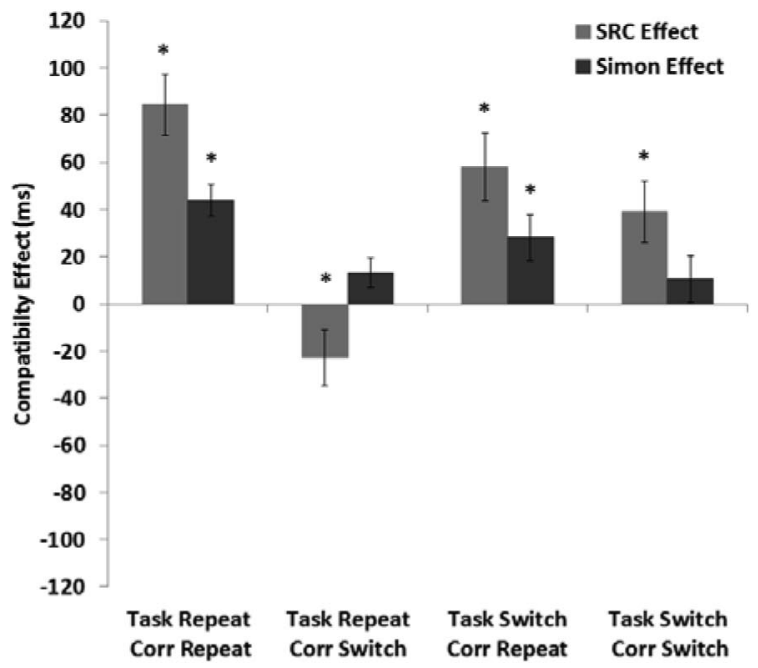

Mostly Incompatible Group

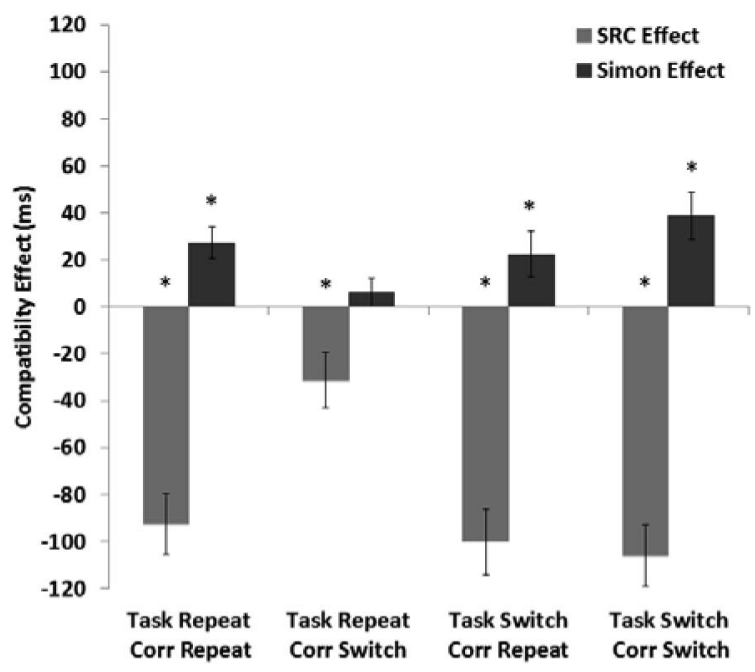

Figure 5. The stimulus-response compatibility (SRC) effect and the Simon effect as a function of task sequence and correspondence sequence for the mostly-compatible and mostly-incompatible groups in Experiment 3 (error bars represent one standard error of means; asterisks indicate significant effects at $\alpha=.05$, as indicated by paired-sample $t$ tests at each data point).

The distributional analysis (see Figure 6) showed interactions of Bin $\times$ Compatibility and of those factors with group, $F \mathrm{~s}(4,184)=$ 15.60 and 5.26, $M S E=199, p$ s $<.001$ and .007 . The Simon effect decreased across the RT distribution for the mostly compatible condition but less so for the mostly incompatible condition. Thus, although the mapping variable did not alter the total Simon effect, it did influence the distributional properties.

$\boldsymbol{P E}$ analysis. There were main effects of task sequence, $F(1$, 46) $=61.54, M S E=18.23, p<.001$, correspondence sequence, $F(1,46)=16.20, M S E=15.62, p<.001$, and correspondence, $F(1,46)=16.78, M S E=28.00, p<.001$. Correspondence interacted with task sequence, $F(1,46)=4.06, M S E=18.03, p<$ .050 , and correspondence sequence, $F(1,46)=15.35, M S E=$ 14.05, $p<.001$. There was also a significant interaction of Correspondence $\times$ Correspondence Sequence $\times$ Task Sequence, $F(1,46)=4.20, M S E=13.35, p<.046$. When the task repeated, the Simon effect was $0.60 \%$ if the S-R correspondence relation repeated and $2.07 \%$ if it switched; when the task switched, the effect was $0.82 \%$ if the correspondence relation repeated and $5.35 \%$ if it switched. Thus, the Simon effect increased when the correspondence relation switched as compared with when it repeated, and the increase was larger when the task switched.

Lastly, the Correspondence $\times$ Correspondence Sequence $\times$ Group interaction was significant, $F(1,46)=5.75, M S E=$ $14.05, p<.021$. For the mostly compatible group, all terms were significant, $F \mathrm{~s}(1,23)>11.65, p \mathrm{~s}<.002$, except the two-way interactions of Task Sequence with Correspondence and with Correspondence Sequence, $F_{\mathrm{s}}(1,23)<1.62, p \mathrm{~s}>.25$. The Simon effect was smaller if the correspondence relation repeated $(0.04 \%)$ than if it switched $(4.87 \%)$, but this difference was larger when the task switched than when it repeated. For the mostly incompatible group, all main effects, $F \mathrm{~s}(1,23)>$

\section{Mostly Compatible Group}

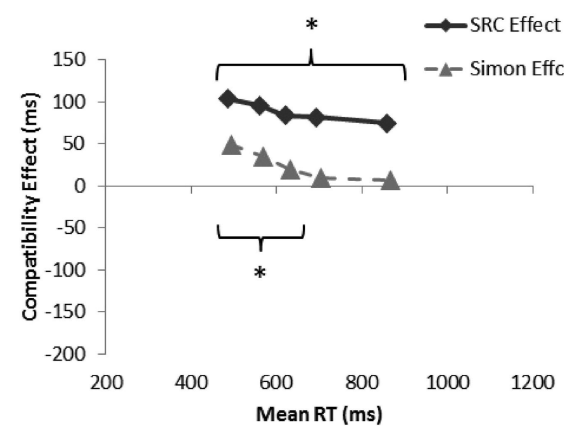

\section{Mostly Incompatible Group}

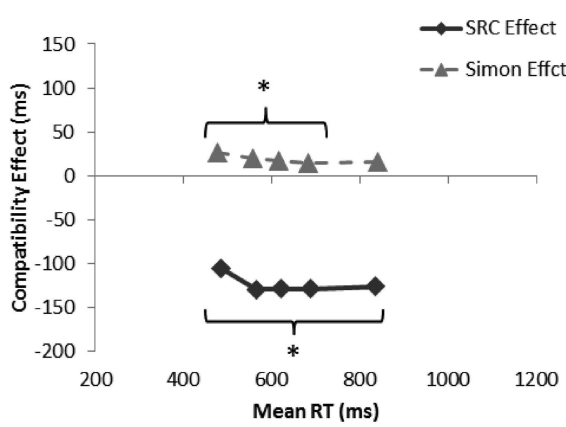

Figure 6. Plots of the stimulus-response compatibility (SRC) effect and the Simon effect against mean response times in the quintile bins in Experiment 3 (effects for the bracketed points, with asterisks, were significant at $\alpha=.05$, as indicated by paired-sample $t$ tests at each data point). 
$5.10, p s<.035$, but no interactions were significant. The Simon effect of $1.94 \%$ did not vary across the correspondence sequence and task sequence conditions. Hence, the increase of the Simon effect for the correspondence-repeat trials was larger for the mostly compatible group than for the mostly incompatible group.

\section{Discussion}

In this experiment, unlike Experiment 2, group had an influence on the SRC and Simon tasks. For the SRC task, the mostly incompatible group showed findings consistent with those of Experiments 1 and 2: The results showed a benefit for the incompatible mapping over the compatible mapping. In contrast, the mostly compatible group showed a benefit for the compatible mapping of smaller absolute size than that shown by the mostly incompatible group. That the SRC effect was negative overall, as it was in Experiment 2 and tended to be in Experiment 1, implies that the bias toward one mapping relation or the other was in addition to the factors that tend to produce a slightly negative SRC effect under unbiased conditions. In agreement with results obtained by $\mathrm{Vu}$ and Proctor (2011) with mixed mappings of the SRC task, this biasing effect produced by making one mapping more frequent than the other also was independent of the factors responsible for the negatively sloped distribution function.

For the Simon task, the interaction of Group $\times$ Correspondence was not significant. The mostly compatible and mostly incompatible groups showed positive Simon effects of similar magnitude. Thus, when the incompatible mapping predominated for the SRC task, causing an increased tendency to make the incompatible response for those trials, this bias did not have much influence on the overall performance of the Simon trials. This result is consistent with the implication that the bias introduced by making compatible or incompatible responses more likely in the SRC task does not influence the strength of the short-term location associations. If it did so, then the manipulation should have had a similar influence on the Simon effect. Although the overall Simon effect was not affected by the mapping bias for the SRC task, the distribution function was. The Simon effect was larger initially and showed a steeper negative slope for the mostly compatible group than for the mostly incompatible group, suggesting that quick responses were in part driven by the mapping bias whereas later ones were not.

Group entered into several higher order interactions for both the SRC task and Simon task, indicating that the sequential effect patterns for both tasks were different when the majority of SRC trials were compatible and when they were incompatible.

\section{General Discussion}

\section{Mean SRC and Simon Effects}

Several findings from the present experiments in which both tasks and mappings were mixed stand out. We first consider those from the SRC task. As demonstrated in Experiment 1, when the two mappings were equally likely, there was no benefit (and, in fact, a slight cost) for the compatible mapping compared with the incompatible mapping on trials for which stimulus location was relevant. This result is in accord with those obtained when the SRC task is performed on all trials, with only the two mappings mixed (e.g., Vu \& Proctor, 2004, 2011), or when trials of the SRC task with one of the location mappings are intermixed with those from a Simon task for which stimulus location is irrelevant (e.g., Proctor $\& \mathrm{Vu}, 2002 \mathrm{~b})$. Thus, the typical advantage for the compatible mapping is also absent in the more complex situation in which both task and mapping decisions must be made.

Second, as demonstrated in Experiment 2, this result did not depend on the relative frequencies of trials for the two tasks. The absence of the SRC effect for trials on which stimulus location was relevant, obtained in Experiment 1 when $50 \%$ of the trials were from the SRC task, was replicated both when $80 \%$ of the trials were from the SRC task and when $20 \%$ of the trials were. This outcome makes sense because either mixed mappings or mixed tasks alone also yield no SRC effect, suggesting that it should not matter whether the majority of trial types are mixed mappings of the SRC task or mixed trials of the Simon task.

In contrast to the results of Experiment 2, manipulation of the percentages of compatible and incompatible SRC trials in Experiment 3 did influence the SRC effect. When $80 \%$ of the trials had a compatible mapping, responses with that mapping were faster than those with the incompatible mapping; the opposite occurred when trials with incompatible mapping were more frequent. This result is in agreement with those obtained when all trials are from the SRC task and the percentages of trials are biased toward one mapping or the other ( $\mathrm{Vu} \&$ Proctor, 2011). Note, though, that averaged across the two bias conditions, the SRC effect was -22 ms. That is, for this unbiased comparison, the incompatible mapping had a slight advantage of shorter RT overall, as it also tended to have in Experiments 1 and 2. Thus, overall, the typical advantage for the compatible spatial mapping effect was again absent, indicating that elimination of the SRC effect under conditions of mixed tasks and/or mappings is a robust phenomenon. The influence of the mapping frequency manipulation on the SRC effect in Experiment 3 appears to be attributable to a separate, intentional bias to respond in a manner consistent with the predominant mapping.

The absence of a positive overall SRC effect in all three experiments is consistent with the view that the long-term associations of the direct route are proactively suppressed when the compatible spatial mapping is applicable on only some trials. However, the negative slopes for the distribution functions for the SRC effect evident in the three experiments suggest that the long-term associations are still playing a role. Although De Jong (1995) favored an explanation of the influence of mixed mappings on the SRC effect in terms of suppression of the direct route, he pointed out that an alternative is that application of the compatible-mapping rule in the indirect route is blocked. If this blocking makes it slightly easier to apply the "respond opposite" rule of the incompatible mapping, then the pattern of results for the SRC task can be explained by assuming that the "suppression" is of the indirect route and that the direct route still produces activation of the corresponding response, which then dissipates $(\mathrm{Vu} \&$ Proctor, 2011).

The results for the Simon task imply even more strongly that the long-term associations of the direct route are still producing activation of the corresponding response in mixed conditions. If proactive suppression were occurring, the Simon effect for the trials on which stimulus location is irrelevant should be absent as well. 
But, Experiment 1 showed a Simon effect that did not differ in size from that of a control condition in which participants performed only the Simon task. The Simon effect in that experiment, as well as in Experiments 2 and 3, also showed the hallmark pattern of decreasing across the RT distribution (see Proctor et al., 2011, for a review). These results imply that activation produced by the long-term associations of the direct route was continuing to affect performance, counter to the prevailing interpretation of the reversal of the Simon effect when the Simon-task trials are mixed with an SRC task for which the mapping for all trials is spatially incompatible (e.g., Marble \& Proctor, 2000). That is, when the biasing effect of the incompatible mapping is "neutralized" by including trials with compatible mapping, the influence of the long-term associations becomes apparent once again.

This finding is similar in nature to one shown by Miles and Proctor (2008) for the impact of "implementation instructions" on the auditory Simon effect. When given an implementation instruction emphasizing a particular noncorresponding spatial relation, such as, "if you hear a low pitch tone on the left side, then press the right button especially fast," the Simon effect was eliminated. However, when in another set of trials an implementation instruction was given that emphasized a particular corresponding relation (e.g., "if you hear a low pitch tone on the right side, then press the right button especially fast"), and the data were averaged across the conditions that emphasized corresponding and noncorresponding relations, thus neutralizing the influences of the instructed relations, the Simon effect was evident in its normal size. Thus, as with implementation instructions, when all trials of the SRC task have incompatible mapping, the reversal of the Simon effect seems to occur by way of the noncorresponding short-term associations established for the SRC task, which produce effects that cover up the activation of the corresponding response being produced by the long-term associations. For comparisons in which the shortterm associations no longer bias a particular response, the contribution of the long-term associations is revealed.

The Simon effect also was uninfluenced in Experiment 2 by whether the SRC task made up $80 \%$ of the trials or the Simon task did. Given that the Simon effect was evident when the Simon task occurred on only $20 \%$ of the trials, it is not too surprising that it also was evident when the Simon task occurred on $80 \%$ of the trials, which is closer to the $100 \%$ value of the standard Simon task. More surprising is that in Experiment 3 the Simon effect was equally evident when $80 \%$ of the SRC trials had an incompatible spatial mapping as when only $20 \%$ did. Thus, although the Simon effect shows a large reversal when $100 \%$ of the mixed SRC trials have an incompatible mapping, no such reversal was obtained when $80 \%$ of the SRC trials were incompatible. This result suggests that the requirement to maintain the compatible mapping in working memory as part of the task set, even if that mapping is applied on only a small portion of trials, is sufficient to nullify the influence of the short-term associations of incompatible locations and reinstate the Simon effect. In other words, the relative strengths of the competing short-term S-R associations are not being influenced by the differences in relative frequency.

Although the dual-route conception offers a straightforward explanation of the dissociation between mean SRC and Simon effects in the present experiments, accounts in terms of stimulus and response coding cannot be ruled out entirely. With regard to stimulus coding, trials for the SRC task included a stimulus (the horizontal or vertical mapping signal) at the center of the display that trials for the Simon task did not. This display difference could have allowed the position of the imperative stimulus to be coded on Simon trials but not on SRC trials. For example, from an attention-shifting perspective (e.g., Rubichi et al., 1997), an argument could be made that attention shifted to the imperative stimulus on Simon trials but remained focused at the center (or shifted back to it) on SRC trials, resulting in a left-right code for spatial position for the Simon task but not the SRC task. Several factors argue against such an account, though. First, when trials of the SRC task with compatible and incompatible mappings are mixed, the SRC effect is eliminated regardless of whether the mapping signal is a centered orientation stimulus (Shaffer, 1965), as in the present study, or a color feature of the imperative stimulus (e.g., Heister \& Schroeder-Heister, 1994; Yamaguchi \& Proctor, 2006). Second, because the SRC effect is eliminated when either mappings or tasks are mixed (and the signal is the color feature), the absence of effect when mappings and tasks together are mixed is a sensible outcome. Finally, the SRC effect tended to decrease across the RT distribution much like the Simon effect did (see, e.g., Figure 2), suggesting that for both trial types the stimulus location codes were producing direct response activation in a similar manner.

With regard to response coding, Ansorge and Wühr (2004) have emphasized that Simon effects (and SRC effects, Wühr, 2011) arise "only when stimulus locations match the top-down selected spatial codes used to discriminate between alternative responses" (p. 365), which they call the response-discrimination account. According to this account, two conditions must be met for Simon and SRC effects to occur: "First, the stimulus-location code must enter WM [working memory]. Second, the WM representation of the $\mathrm{S}-\mathrm{R}$ rules must include a response-location code" (Wühr \& Bieble, 2011, p. 444). The use of left and right keypress responses in the present experiments satisfies the second condition, so any explanation of the results derived from the response-discrimination account must rely on the first condition, reduced "entry" (i.e., activation) of the stimulus-location code in working memory. Note that this restriction rules out response discrimination per se as the basis of the present results.

In separate studies of the Simon and SRC tasks, Wühr and Biebl (2011) and Wühr (2011) showed that a high working memory load can eliminate or reduce the Simon and SRC effects. A verbal memory load of five consonants reduced both effects, whereas a visual memory load of four filled locations in matrix reduced the SRC effect but eliminated the Simon effect entirely. In extending the response-discrimination account to spatial SRC, Wühr noted that two sources of the effect could be assumed, S-R translation and S-R priming. He indicated that, on this account, "WM [working memory] load could modulate the spatial-mapping effect by interfering with S-R translation or by interfering with S-R priming" (p. 403), and concluded that his results were consistent only with the latter priming account. Lack of priming of the corresponding response attributable to high memory load could explain the absence of SRC effect in the current experiments, but the presence of the Simon effect in the present experiments indicates that the stimulus-location code was indeed "priming" the corresponding response. Thus, to maintain a memory load interpretation of the present data, an assumption would have to be made that stimulus- 
location information was entering working memory and priming responses for trials on which it was irrelevant but not for trials on which it was relevant. Note that this enigma is the same as that encountered by the proactive suppression hypothesis, of which the load account is a variant.

\section{Sequential Effects}

Trial sequences in all experiments were analyzed in terms of task-repetition/switch and correspondence-repetition/switch. In Experiments 1 and 2, the results were straightforward. Task repetition and correspondence repetition benefited RT, with the benefit being largest when both repeated. With regard to the SRC and Simon effects, though, the primary sequential factor was correspondence repetition/switch. Both effects were larger when the spatial correspondence from the previous trial repeated compared with when it switched, and this result did not depend on whether the task repeated or switched, or in Experiment 2, on which task, SRC or Simon, occurred on $80 \%$ of the trials. Thus, whatever mechanism underlies the sequential modulations of the SRC and Simon effects (e.g., reactive suppression of the direct route, priming or inhibition of an S-R mapping rule), this mechanism carried over between the two tasks. Nevertheless, the SRC effect was absent overall or considerably reduced in these experiments, and the sequential effects from the Simon trials had relatively minor impacts on the SRC trials.

The results of Experiment 3 were more complicated. For the SRC task, the absolute size of the SRC effect (positive for the mostly compatible group and negative for the mostly incompatible group) was larger when the correspondence relation repeated than when it switched, but mainly for trials on which the task also repeated. The sequential modulation of the Simon effect did not carry over much to the SRC trials, implying that there is priming of an S-R mapping specific to SRC trials. If reactive inhibition of the direct route were the cause of the sequential effect in the SRC trials, it should have transferred from the Simon trials to the SRC trials as well.

For the Simon task, the mostly compatible group showed results similar to those of Experiments 1 and 2: The Simon effect was larger when the correspondence relation repeated than when it switched, and this did not depend on whether the task repeated or switched, again suggesting a common mechanism underlying sequential effects in the two tasks. However, for the mostly incompatible group, task repetition/switch did matter: When the task repeated, the Simon effect was larger if the correspondence relation also repeated than if it switched; when the task switched, the influence of correspondence relation was larger if it also switched. Note that because $80 \%$ of the SRC trials for this group had incompatible mapping, the Simon trials were more likely to follow a trial with incompatible mapping than one with compatiblemapping when the task switched. Thus, the larger Simon effect after task switches can be attributed to the bias toward the incompatible mapping carrying over from the prior SRC trial. An implication is that the sequential modulations of the SRC and Simon effects involve different mechanisms. If the modulations of these effects were attributable solely to a common mechanism, the sequential effect should not depend on whether the prior task was the same or different from the current task. On the whole, it appeared that the sequential modulations could carry over from the
SRC trials to the Simon trials throughout the three experiments, and the modulations depended on whether the Simon trial followed a compatible or incompatible mapping trial. These outcomes are consistent with prior studies for which task-defined S-R mappings reversed the Simon effect (e.g., Hedge \& Marsh, 1975). In contrast, the sequential modulations for the Simon trials had relatively little impact on the SRC trials. If sequential modulation of the Simon effect is attributable to reactive suppression of long-term associations, the suppression did not carry over to the SRC effect, indicating that the mechanism underlying the SRC effect is distinct from that underlying the Simon effect (or, automatic response activation). The results are consistent with the view that the SRC effect is attributable mainly to S-R translation of the indirect route (e.g., Fitts \& Deininger, 1954).

In previous studies of the Simon task, effects of trial sequences on the Simon effect were analyzed according to whether the preceding trial was spatially corresponding or not (e.g., Soetens et al., 2010), rather than whether the spatial relation repeated or not. A typical finding is that the Simon effect is reduced, and often absent, after a noncorresponding trial, which many researchers have attributed to the direct route being reactively inhibited. Analysis of the RT data in terms of correspondence of the preceding trial, correspondence of the current trial, and task sequence showed a similar three-way interaction in all experiments. For task-repeat trials, large positive Simon effects were obtained after trials for which the relation on the prior trial had been corresponding and large negative Simon effects after trials for which the previous relation had been noncorresponding. These differences were attenuated for task-switch trials, for which location had been relevant on the previous trial. In neither Experiment 2 nor 3 did this pattern interact with group, indicating that sequential changes of the Simon effect for trials on which the Simon task repeats are largely independent of the proportions of SRC and Simon trials and proportions of compatible and incompatible SRC mappings.

This sequential analysis also suggests that the correspondence in the preceding SRC trial influences the Simon effect in the current trial, consistent with the previous analysis. However, the influence of the prior S-R relation was larger when the preceding trial was from the Simon task. In fact, the Simon effect was consistently negative throughout the three experiments if the current trial followed a noncorresponding trial. Because suppression of the direct route can only eliminate the Simon effect, something more than reactive suppression of the direct route must be operating to reverse the effect, such as negative priming of a compatible response.

\section{Conclusion}

The main findings of the present study are as follows. (a) The SRC and Simon effects are dissociable; the SRC effect was absent overall throughout the three experiments, but the Simon effect was present. (b) The SRC and Simon effects were larger at short RT bins and decreased at longer RT bins, suggesting that the direct activation route is not suppressed but may be inhibited in a reactive fashion. This conclusion contradicts the proactive suppression account of the elimination of the SRC and Simon effects favored in prior studies in which tasks or mappings were mixed. (c) The SRC effect is influenced relatively little by the correspondence relation of a prior Simon-task trial, suggesting that the SRC effect 
involves more than long-term associations (e.g., bias of the compatible or incompatible mapping rules, or stronger short-term associations, which depends on the relative frequency of the mappings). (d) The Simon effect is modulated by the correspondence relation of a prior SRC trial, suggesting that the Simon and SRC effects have a process in common.

The presence of the Simon effect in the present experiments implies that the direct response-activation route is not suppressed proactively under mixed conditions but rather that the activation it produces may be inhibited reactively (Vu \& Proctor, 2011). The elimination of the Simon effect when Simon-task trials were mixed with incompatibly mapped SRC trials alone in other studies (e.g., Marble \& Proctor, 2000) likely is a consequence of bias toward the spatially incompatible response in the intentional, indirect response-selection route.

The results of the sequential analysis are consistent with these conclusions. The SRC effect was modulated by the correspondence relation on the previous trial, but the modulation tended to be smaller or even absent when the previous trial was from the Simon task, suggesting that the sequential effect relies primarily on the bias of the S-R mapping (in the intentional route). In contrast, the influence of the correspondence relation from the previous trial fully propagated to the Simon effect, regardless of whether the prior trial was from the Simon or SRC task. This result suggests that sequential modulation of the SRC effect depends mainly on task-defined rules of the indirect, intentional routes but not on reactive inhibition of the direct route, whereas sequential modulation of the Simon effect depends on reactive inhibition of the direct route when the trial follows the Simon task, and on transfer of the task-defined spatial mappings when the trial follows the SRC task. Hence, the Simon effect is determined by both the direct and indirect routes and the SRC effect mainly by the indirect, intentional route.

Therefore, these present findings are inconsistent with the most widely accepted previous explanation of the modulations of the Simon and SRC effects in the mixed-task or -mapping conditions, which attributes the modulations mainly to proactive suppression of the direct route. Sequential changes of the Simon effect may reflect reactive inhibition of activation occurring through the direct route, but the overall elimination of the SRC effect in mixed-task conditions is a result of bias in the application of mapping rules and not proactive suppression of the direct route.

\section{References}

Ansorge, U., \& Wühr, P. (2004). A response-discrimination account of the Simon effect. Journal of Experimental Psychology: Human Perception and Performance, 30, 365-377. doi:10.1037/0096-1523.30.2.365

De Jong, R. (1995). Strategical determinants of compatibility effects with task uncertainty. Acta Psychologica, 88, 187-207. doi:10.1016/00016918(94)E0067-P

De Jong, R., Liang, C., \& Lauber, E. (1994). Conditional and unconditional automaticity: A dual-process model of effects of spatial stimulusresponse correspondence. Journal of Experimental Psychology: Human Perception and Performance, 20, 731-750. doi:10.1037/0096-1523.20 .4 .731

Dutta, A., \& Proctor, R. W. (1992). Persistence of stimulus-response compatibility effects with extended practice. Journal of Experimental Psychology: Learning, Memory, and Cognition, 18, 801-809. doi: 10.1037/0278-7393.18.4.801
Egner, T. (2008). Multiple conflict-driven control mechanisms in the human brain. Trends in Cognitive Sciences, 12, 374-380. doi:10.1016/ j.tics.2008.07.001

Fitts, P. M., \& Deininger, R. L. (1954). S-R compatibility: Correspondence among paired elements within stimulus and response codes. Journal of Experimental Psychology, 48, 483-492. doi:10.1037/h0054967

He, X., Fan, S., Zhou, K., \& Chen, L. (2004). Cue validity and object-based attention. Journal of Cognitive Neuroscience, 16, 1085-1097. doi: 10.1162/0898929041502689

Hedge, A., \& Marsh, N. W. A. (1975). The effect of irrelevant spatial correspondences on two-choice response time. Acta Psychologica, 39, 427-439. doi:10.1016/0001-6918(75)90041-4

Heister, G., \& Schroeder-Heister, P. (1994). Spatial S-R compatibility: Positional instructions vs. compatibility instruction. Acta Psychologica, 85, 15-24. doi:10.1016/0001-6918(94)90017-5

Hommel, B. (2011). The Simon effect as tool and heuristic. Acta Psychologica, 136, 189-202. doi:10.1016/j.actpsy.2010.04.011

Hommel, B., Proctor, R. W., \& Vu, K.-P. L. (2004). A feature-integration account of sequential effects in the Simon task. Psychological Research/ Psychologische Forschung, 68, 1-17. doi:10.1007/s00426-003-0132-y

Iani, C., Rubichi, S., Gherri, E., \& Nicoletti, R. (2009). Co-occurrence of sequential and practice effects in the Simon task: Evidence for two independent mechanisms affecting response selection. Memory \& Cognition, 37, 358-367. doi:10.3758/MC.37.3.358

Kornblum, S., Hasbroucq, T., \& Osman, A. (1990). Dimensional overlap: Cognitive basis for stimulus-response compatibility - A model and taxonomy. Psychological Review, 97, 253-270. doi:10.1037/0033-295X.97 .2 .253

Marble, J. G., \& Proctor, R. W. (2000). Mixing location-relevant and location-irrelevant trials in choice-reaction tasks: Influences of location mapping on the Simon effect. Journal of Experimental Psychology: Human Perception and Performance, 26, 1515-1533. doi:10.1037/ 0096-1523.26.5.1515

Miles, J. D., \& Proctor, R. W. (2008). Improving performance through implementation intentions: Are preexisting response biases replaced? Psychonomic Bulletin \& Review, 15, 1105-1110. doi:10.3758/PBR.15 .6 .1105

Moresi, S., Adam, J. J., Rijcken, J., \& Van Gerven, P. W. M. (2008). Cue validity effects in response preparation: A pupillometric study. Brain Research, 1196, 94-102. doi:10.1016/j.brainres.2007.12.026

Proctor, R. W., \& Fisicaro, S. A. (1977). Time, capacity, and selection between perceptual attributes. Journal of Experimental Psychology: Human Perception and Performance, 3, 337-348. doi:10.1037/00961523.3.2.337

Proctor, R. W., \& Lu, C.-H. (1999). Processing irrelevant information: Practice and transfer effects in choice-reaction tasks. Memory \& Cognition, 27, 63-77. doi:10.3758/BF03201214

Proctor, R. W., Miles, J. D., \& Baroni, G. (2011). Reaction-time distribution analysis of spatial correspondence effects. Psychonomic Bulletin \& Review, 18, 242-266. doi:10.3758/s13423-011-0053-5

Proctor, R. W., \& Vu, K.-P. L. (2002a). Eliminating, magnifying, and reversing spatial compatibility effects with mixed location-relevant and irrelevant trials. In W. Prinz, \& B. Hommel (Eds.), Common mechanisms in perception and action: Attention and performance, (Vol. XIX, pp. 443-473). Oxford, UK: Oxford University Press.

Proctor, R. W., \& Vu, K.-P. L. (2002b). Mixing location-irrelevant and location-relevant trials: Influence of stimulus mode on spatial compatibility effects. Memory \& Cognition, 30, 281-293. doi:10.3758/ BF03195289

Proctor, R. W., \& Vu, K.-P. L. (2006). Stimulus-response compatibility principles: Data, theory, and application. Boca Raton, FL: CRC Press.

Proctor, R. W., Vu, K.-P. L., \& Marble, J. G. (2003). Spatial compatibility effects are eliminated when intermixed location-irrelevant trials produce 
the same spatial codes. Visual Cognition, 10, 15-50. doi:10.1080/ 713756673

Ridderinkhof, K. R. (2002). Activation and suppression in conflict tasks: Empirical clarification through distributional analyses. In W. Prinz, \& B. Hommel (Eds.), Common mechanisms in perception and action: Attention and performance XIX (pp. 494-519). New York, NY: Oxford University Press.

Rubichi, S., Nicoletti, R., Iani, C., \& Umiltà, C. (1997). The Simon effect occurs relative to the direction of an attention shift. Journal of Experimental Psychology: Human Perception and Performance, 23, 13531364. doi:10.1037/0096-1523.23.5.1353

Shaffer, L. H. (1965). Choice reaction with variable S-R mapping. Journal of Experimental Psychology, 70, 284-288. doi:10.1037/h0022207

Simon, J. R. (1990). The effects of an irrelevant directional cue on human information processing. In R. W. Proctor, \& T. G. Reeve (Eds.), Stimulus-response compatibility: An integrated perspective (pp. 31-86). Amsterdam, The Netherlands: North-Holland.

Soetens, E., Maetens, K., \& Zeischka, P. (2010). Practice-induced and sequential modulations of the Simon effect. Attention, Perception, \& Psychophysics, 72, 895-911. doi:10.3758/APP.72.4.895

Spironelli, C., Tagliabue, M., \& Umiltà, C. (2009). Response selection and attention orienting: A computational model of Simon effect asymmetries. Experimental Psychology, 56, 274-282. doi:10.1027/1618-3169 .56 .4 .274

Sturmer, B., Leuthold, H., Soetens, E., Schröter, H., \& Sommer, W. (2002). Control over location-based response activation in the Simon task: Behavioral and electrophysiological evidence. Journal of Experimental Psychology: Human Perception and Performance, 28, 1345-1363. doi: 10.1037/0096-1523.28.6.1345

Vu, K.-P. L., \& Proctor, R. W. (2004). Mixing compatible and incompatible mappings: Elimination, reduction, and enhancement of spatial compatibility effects. Quarterly Journal of Experimental Psychology A: Human Experimental Psychology, 57, 539-556.
Vu, K.-P. L., \& Proctor, R. W. (2008). Age differences in response selection for pure and mixed stimulus-response mappings and tasks. Acta Psychologica, 129, 49-60. doi:10.1016/j.actpsy.2008.04.006

Vu, K.-P. L., \& Proctor, R. W. (2011). Influence of bias on visuospatial and verbal compatibility effects in mixed mapping environments. Attention, Perception, \& Psychophysics, 73, 2594-2605. doi:10.3758/s13414-0110205-8

Wendt, M., Kluwe, R. H., \& Peters, A. (2006). Sequential modulations of interference evoked by processing task-irrelevant stimulus features. Journal of Experimental Psychology: Human Perception and Performance, 32, 644-667. doi:10.1037/0096-1523.32.3.644

Wuhr, P. (2011). Working-memory load decreases mappings effects in spatial-compatibility tasks. Psychological Research/Psychologische Forschung, 75, 393-405. doi:10.1007/s00426-010-0317-0

Wuhr, P., \& Ansorge, U. (2007). A Simon effect in memory retrieval: Evidence for the response-discrimination account. Psychonomic Bulletin \& Review, 14, 984-988. doi:10.3758/BF03194132

Wuhr, P., \& Biebl, R. (2011). The role of working memory in spatial S-R correspondence effects. Journal of Experimental Psychology: Human Perception and Performance, 37, 442-454. doi:10.1037/a0020563

Yamaguchi, M., \& Proctor, R. W. (2006). Stimulus-response compatibility with pure and mixed mappings in a flight task environment. Journal of Experimental Psychology: Applied, 12, 207-222. doi:10.1037/1076898X.12.4.207

Yamaguchi, M., \& Proctor, R. W. (2012). Multidimensional vector model of stimulus-response compatibility. Psychological Review, 119, 272303. doi: $10.1037 / \mathrm{a} 0026620$

Zorzi, M., \& Umiltà, C. (1995). A computational model of the Simon effect. Psychological Research/Psychologische Forschung, 58, 193 205. doi:10.1007/BF00419634

Received March 25, 2012

Revision received July 9, 2012

Accepted July 17, 2012 